\title{
AAV capsid variants with brain-wide transgene expression and decreased liver targeting after intravenous delivery in mouse and marmoset
}

\author{
David Goertsen ${ }^{1,12}$, Nicholas C. Flytzanis ${ }^{1,12}$, Nick Goeden 1,12, Miguel R. Chuapoco ${ }^{1}$, \\ Alexander Cummins ${ }^{2}$, Yijing Chen ${ }^{3}$, Yingying Fan ${ }^{3}$, Qiangge Zhang ${ }^{4,5}$, Jitendra Sharma ${ }^{4,6,7,8}$,

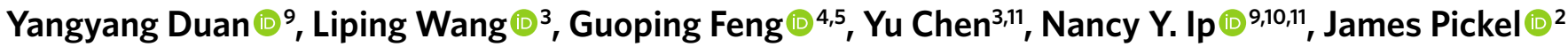 \\ and Viviana Gradinaru $1{ }^{1} \otimes$
}

\begin{abstract}
Genetic intervention is increasingly being explored as a therapeutic option for debilitating disorders of the central nervous system. The safety and efficacy of gene therapies rely upon expressing a transgene in affected cells while minimizing off-target expression. Here we show organ-specific targeting of adeno-associated virus (AAV) capsids after intravenous delivery, which we achieved by employing a Cre-transgenic-based screening platform and sequential engineering of AAV-PHP.eB between the surface-exposed AA452 and AA460 of VP3. From this selection, we identified capsid variants that were enriched in the brain and targeted away from the liver in C57BL/6J mice. This tropism extends to marmoset (Callithrix jacchus), enabling robust, non-invasive gene delivery to the marmoset brain after intravenous administration. Notably, the capsids identified result in distinct transgene expression profiles within the brain, with one exhibiting high specificity to neurons. The ability to cross the blood-brain barrier with neuronal specificity in rodents and non-human primates enables new avenues for basic research and therapeutic possibilities unattainable with naturally occurring serotypes.
\end{abstract}

$\mathrm{T}$ he wide array of debilitating disorders affecting the central nervous system (CNS) make it a primary target for the application of novel therapeutic modalities, as evidenced by the rapid development and implementation of gene therapies targeting the brain. AAVs are increasingly used as delivery vehicles owing to their strong clinical safety record, low pathogenicity and stable, non-integrating expression in vivo ${ }^{1}$. AAVs were first approved for gene therapy in humans in 2012 to treat lipoprotein lipase deficiency ${ }^{2}$ and have, more recently, been approved to treat spinal muscular atrophy ${ }^{3}$, retinal dystrophy and hemophilia $a^{4,5}$. Many more advanced-stage clinical trials using AAVs are underway ${ }^{6}$. Currently, most AAV-based gene therapies rely on naturally occurring serotypes with highly overlapping tropisms ${ }^{7}$, limiting the applicability, efficacy and safety of novel gene therapies. At the same time, the inability to broadly and efficiently target many therapeutically relevant cell populations within organs that have traditionally been refractory to AAV delivery has hindered novel exploratory and therapeutic efforts. These constraints, which are especially prevalent for the CNS, motivated us to enhance AAV efficiency and specificity through directed evolution.

Naturally occurring AAVs have evolved to broadly infect cells ${ }^{8}$, which is a desirable characteristic for the survival of the virus but undesirable for targeting specific cell types. This limitation has typically been addressed by injecting viral vectors directly into the area of interest in the $\mathrm{CNS}^{9}$, through either intracranial or intrathecal injection. Although direct injection is a valuable technique for targeting focal cell populations, it requires considerable surgical expertise, and this approach cannot address applications that require broad and uniform area coverage (for example, all cortex or all striatum) or applications that are surgically difficult to access owing to the invasiveness of direct injections (for example, cerebellum and dorsal raphe ${ }^{9-11}$. Systemic administration into the bloodstream is an appealing solution to the limitations of direct injections, as gene therapy vectors can be non-invasively delivered throughout the body. However, naturally occurring AAV serotypes tend to target non-CNS tissues, notably the liver, at high levels ${ }^{7,8}$. The liver is an immunologically active organ, with large populations

'Division of Biology and Biological Engineering, California Institute of Technology, Pasadena, CA, USA. ${ }^{2}$ National Institute of Mental Health, National Institutes of Health, Bethesda, MD, USA. ${ }^{3}$ Chinese Academy of Sciences Key Laboratory of Brain Connectome and Manipulation, Shenzhen Key Laboratory of Translational Research for Brain Diseases, The Brain Cognition and Brain Disease Institute, Shenzhen Institute of Advanced Technology, Chinese Academy of Sciences; Shenzhen-Hong Kong Institute of Brain Science-Shenzhen Fundamental Research Institutions, Shenzhen, China. ${ }^{4}$ McGovern Institute for Brain Research, Department of Brain and Cognitive Sciences, Massachusetts Institute of Technology, Cambridge, MA, USA. ${ }^{5}$ Stanley Center for Psychiatric Research, Broad Institute of MIT and Harvard, Cambridge, MA, USA. ${ }^{6}$ Picower Institute of Learning and Memory, Massachusetts Institute of Technology, Cambridge, MA, USA. ${ }^{7}$ Tan \& Yang Center for Autism Research, McGovern Institute of Brain Research, Massachusetts Institute of Technology, Cambridge, MA, USA. ${ }^{8}$ Athinoula A. Martinos Center for Biomedical Imaging, Department of Radiology, Massachusetts General Hospital and Harvard Medical School, Boston, MA, USA. ${ }^{9}$ Division of Life Science, State Key Laboratory of Molecular Neuroscience and Molecular Neuroscience Center, Hong Kong University of Science and Technology, Clear Water Bay, Kowloon, Hong Kong, China. ${ }^{10}$ Hong Kong Center for Neurodegenerative Diseases, Hong Kong Science Park, Hong Kong, China. "Guangdong Provincial Key Laboratory of Brain Science, Disease and Drug Development, Shenzhen-Hong Kong Institute of Brain Science, HKUST Shenzhen Research Institute, Shenzhen, Guangdong, China. ${ }^{2}$ These authors contributed equally: David Goertsen, Nicholas C. Flytzanis, Nick Goeden. 凶e-mail: viviana@caltech.edu 


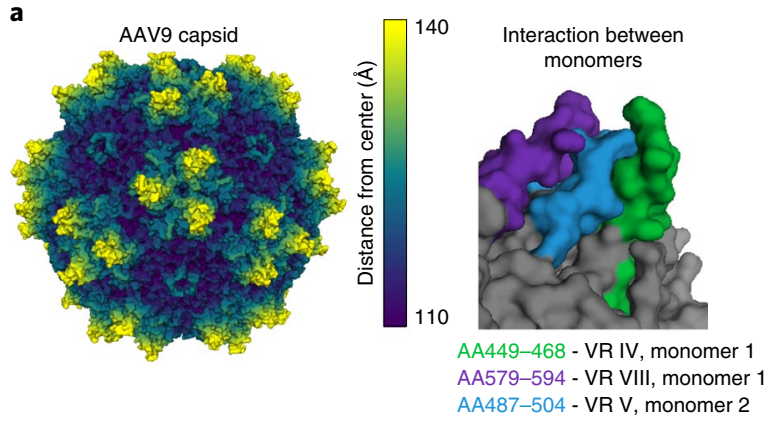

b

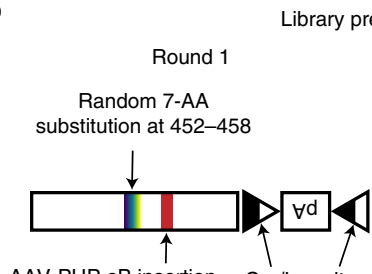

AAV-PHP.eB insertion Cre/Lox sites

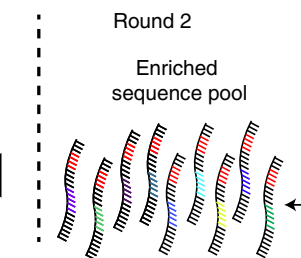

Cre recombination in vivo

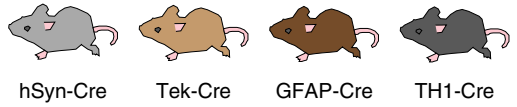

Tissue harvesting

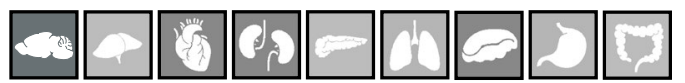

NGS and enrichment determination

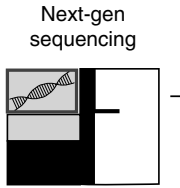

Compare across Cre transgenics

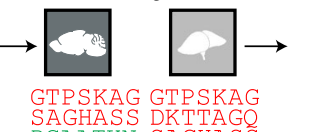

Select variants SAGHASS DKTTAG DGAATKN

After round 2
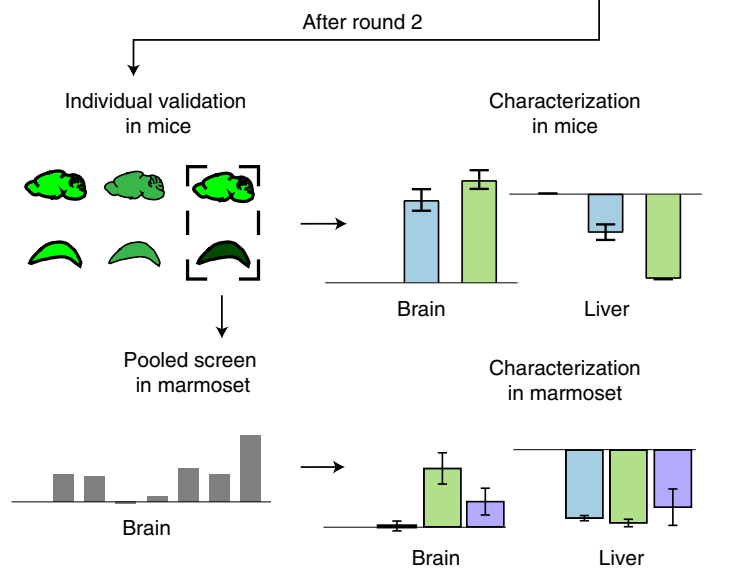

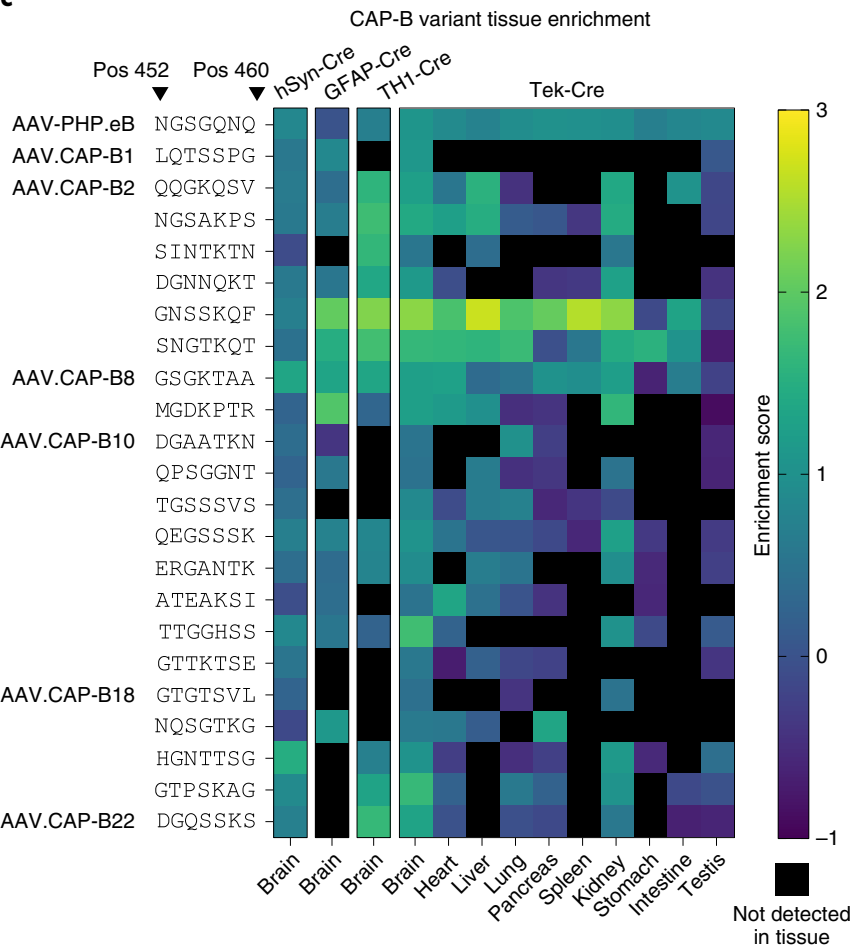

d

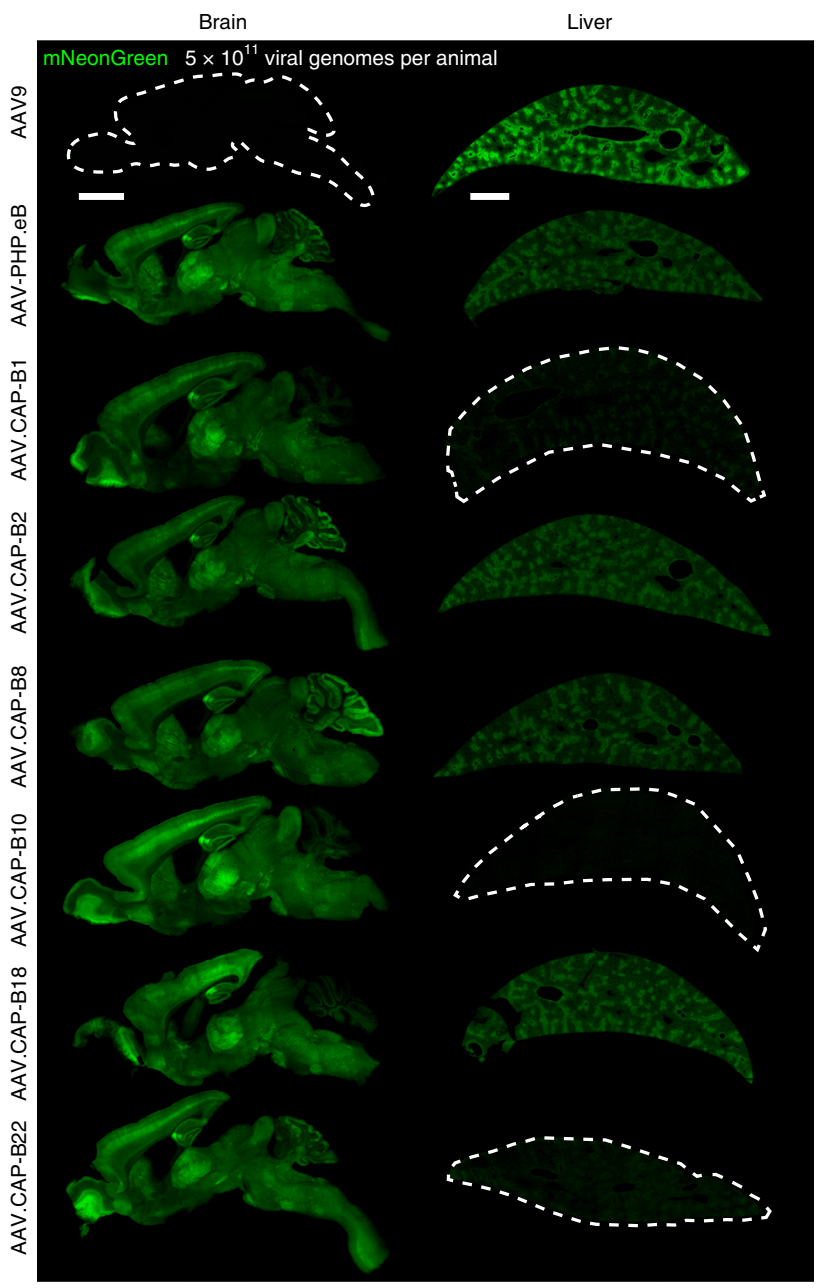


Fig. 1 | Capsid engineering locations and CAP-B library characterization. a, Left, AAV9 capsid surface model illustrates the location of the protruding loop structures, in yellow. Right, capsid zoom-in shows the spike created by the AA579-AA594 and the AA449-AA468 variable regions of one AAV9 monomer interacting with the AA487-AA504 variable region of a second monomer. $\mathbf{b}$, Experimental workflow. First, a library of variants is designed by mutating AAs between the AA452 and AA458 sites on an AAV-PHP.eB backbone. Cre-Lox sites are inserted into the viral genome to enable detection of genomes that reach Cre-recombinase-containing cells. This viral library is used to create viral capsids containing their corresponding genome. Next, variants are injected into transgenic mouse lines, where Cre recombination occurs after transduction. After 3 weeks of expression, relevant tissues are harvested from each transgenic line, and the DNA is isolated, amplified and prepared for sequencing. After NGS, variants are ranked based on enrichment in select tissues, and the top 10-20\% performers repeat the selection process above. Top enriched variants with unique tissue tropism profiles after two rounds of selection are validated individually for transgene expression by in vivo screening in mice. Variants that display similar brain, but reduced liver, transgene expression are selected for pooled marmoset testing. c, Heat map plotting the enrichment scores of a subset of the 22 variants identified after two rounds of selection in vivo across Cre transgenic lines shows enrichment and specificity for the CNS. The sequences for the 7-AA substitutions present in each of the variants show divergence from the parent AAV-PHP.eB sequence (see also Extended Data Fig. 1). d, mNeonGreen was packaged in each variant under control of the ubiquitous CAG promoter and intravenously administered to mice at a dose of $5 \times 10^{11}$ viral genomes per animal. Transgene expression was assayed by mNeonGreen fluorescence throughout brain and liver. The expression from ssAAV9, ssAAV-PHP.eB, ssAAV.CAP-B1, ssAAV.CAP-B2, ssAAV.CAP-B8, ssAAV.CAP-B10, ssAAV.CAP-B18 and ssAAV.CAP-B22 packaging CAG-mNeonGreen as assessed after 2 weeks. Direct comparison of the expression profiles in brain and liver of the top-performing variants shows a correlation between validated tropisms and those predicted by the NGS data. Scale bars, $2 \mathrm{~mm}$.

of phagocytic cells that play a critical role in immune activation ${ }^{12,13}$. Viral targeting to these tissues can trigger immune response, such as liver toxicity ${ }^{14,15}$, reducing the safety and limiting the efficacy of systemic injection. Naturally occurring serotypes also have severely limited transduction efficiency in the brain owing to the stringency of the blood-brain barrier, consequently requiring the production of large, high-quality vector titers. Enabling and refining both direct and systemic injections will best equip the community with flexible options to pursue a broader range of neuroscience applications.

To target a precise anatomical region and/or cell type in conjunction with systemic injection, specificity can be obtained or refined through the inclusion of cell-type-specific promoters ${ }^{16-18}$, enhancer elements ${ }^{19-23}$ and microRNA target sites ${ }^{24,25}$ into AAV viral genomes, or viral capsids can be engineered to alter their tissue tropism. Previously, we harnessed the power and specificity of Cre transgenic mice to apply increased selective pressure to viral engineering, leading to variants AAV-PHP.B and AAV-PHP.eB, which cross the blood-brain barrier after intravenous administration and broadly transduce cells throughout the $\mathrm{CNS}^{22,26}$. These variants transformed the way that the CNS is studied in rodents, opening up completely novel approaches for measuring and affecting brain activity, in both exploratory and translational contexts ${ }^{18,27-29}$. However, these variants also transduce cells in the liver and other off-target organs. Therefore, the development of AAV variants with decreased liver targeting is particularly important to avoid strong, systemic immune responses ${ }^{30-32}$.

To achieve specificity with the viral capsid, it is imperative that one applies both positive and negative selective pressure to engineered capsid libraries. The M-CREATE method ${ }^{33}$ applies next-generation sequencing (NGS) of synthetic libraries with built-in controls to screen viral variants across multiple Cre transgenic lines for both positive and negative features. Using the M-CREATE method, we selected a panel of novel variants that are both highly enriched in the CNS and targeted away from varying peripheral organs. Transgene expression after delivery with AAV.CAP-B10, described herein, was found to be highly specific for neurons in the CNS, significantly decreased in all peripheral organs assayed and targeted away from the liver in mice. Notably, although AAV-PHP.B failed to translate to non-human primates (NHPs) ${ }^{34,35}$, here we show robust transgene expression after intravenous administration of newly engineered variants in the adult marmoset CNS with minimal liver expression compared to AAV9 and AAV-PHP.eB.

\section{Results}

Engineering AAV capsids at the three-fold point of symmetry. The most commonly altered position within the AAV capsid is the surface-exposed loop containing amino acid (AA) 588, because it is the site of heparan sulfate binding in AAV2 (ref. ${ }^{36}$ ) and is

Fig. 2 | AAV.CAP-B10 tissue expression profile is biased toward the brain, with a significant decrease in liver targeting. a, ssAAV9, ssAAV-PHP.eB and ssAAV.CAP-B10, packaging a nuclear-localized GFP under the control of the CAG promoter, were intravenously injected into male adult mice at $1 \times 10^{11}$ viral genomes per mouse. GFP fluorescence was assessed after three weeks of expression. Quantification of the total number of cells expressing GFP in the brain $(P=0.0016$ (AAV9 versus AAV-PHP.eB), $P=0.0003$ (AAV9 versus AAV.CAP-B10) and $P=0.4345$ (AAV-PHP.eB versus AAV.CAP-B10)) and the average brightness per cell ( $P=0.06$ (AAV9 versus AAV-PHP.eB), $P=0.0043$ (AAV9 versus AAV.CAP-B10) and $P>0.999$ (AAV-PHP.eB versus AAV.CAP-B10)) show an increase in transgene expression efficiency in the brain of AAV.CAP-B10 similar to AAV-PHP.eB. $\mathbf{b}$, Quantification of the total percentage of cells expressing GFP in the liver ( $P=0.0127$ (AAV9 versus AAV-PHP.eB), $P<0.0001$ (AAV9 versus AAV.CAP-B10) and $P=0.0047$ (AAV-PHP.eB versus AAV. CAP-B10)) and average brightness per cell $(P=0.080$ (AAV9 versus AAV-PHP.eB), $P=0.0009$ (AAV9 versus AAV.CAP-B10) and $P=0.48(A A V-P H P . e B$ versus AAV.CAP-B10)) show an iterative decrease in transgene expression efficiency from AAV-PHP.eB to AAV.CAP-B10. a, b, $n=6$ mice per group, mean \pm s.e. Statistical significance was determined using Brown-Forsythe and Welch ANOVA tests with the Dunnett T3 correction for multiple comparisons for transgene expression and the Kruskal-Wallis test with Dunn's correction for multiple comparisons for brightness. c, d, Within the brain, AAV.CAP-B10 is biased toward neurons ( $P=0.44$ (cortex), $P=0.21$ (hippocampus), $P=0.81$ (thalamus), $P=0.56$ (striatum), $P=0.31$ (midbrain) and $P=0.82$ (total)), stained with $\alpha$ NeuN (Abcam, 177487). Expression and percentage of neurons expressing transgene. e, $\mathbf{f}$, Expression and percentage of astrocytes expressing transgene $(P=0.00002$ (cortex), $P=0.0034$ (hippocampus), $P=0.017$ (thalamus), $P=0.057$ (striatum),$P=0.0070$ (midbrain) and $P=0.0012$ (total)), stained with $\alpha \mathrm{S} 100$ (Abcam, 868). $\mathbf{g}$, h, Expression and percentage of oligodendrocytes expressing transgene $(P=0.0039$ (cortex), $P=0.00046$ (hippocampus), $P=0.0047$ (thalamus), $P=0.0085$ (striatum), $P=0.00019$ (midbrain) and $P=0.0018$ (total)), stained with $\alpha$ Olig2 (Abcam, 109186). c-h, $n=6$ mice per group, except for hippocampal $\mathrm{NeuN}^{+}$counts of AAV.CAP-B10, where $n=3$, mean \pm s.e. Statistical significance was determined using two-sided Welch's $t$-tests. The contribution of cells from different classification to overall EGFP expression was measured and indicates a shift toward neuronal specificity of AAV.CAP-B10 compared to AAV-PHP.eB (see also Extended Data Figs. 2-4). Scale bars, $200 \mu$ m. A.U., arbitray units; NS, not significant; vg, viral genomes. 
amenable to peptide display ${ }^{37,38}$. The only known receptors for AAV9 are $\mathrm{N}$-linked terminal galactose ${ }^{39}$ and the AAV receptor ${ }^{40,41}$, but the possibility of co-receptors is still unexplored. Binding interactions with cell surface receptors occur near the three-fold axis of symmetry of the viral capsid (Fig. 1a) where the surface-exposed loop containing AA455 of AAV9 is the farthest protruding ${ }^{42}$. Having already engineered AAV-PHP.B and AAV-PHP.eB at the AA588 loop for enhanced CNS transduction ${ }^{22,26}$, and with the additional goal of decreasing viral transduction of peripheral organs, we theorized that introducing diversity into the AA455 loop would enhance the interaction with existing mutations of the AA588 loop and refine transduction. Thus, we engineered and performed two rounds of selection with a 7-AA substitution library of the AA455 loop, between AA452 and AA460 (Fig. 1a) in AAV-PHP.eB.

We applied the M-CREATE method developed by Kumar et al. ${ }^{33}$ to identify AAV variants with desired tropism after systemic

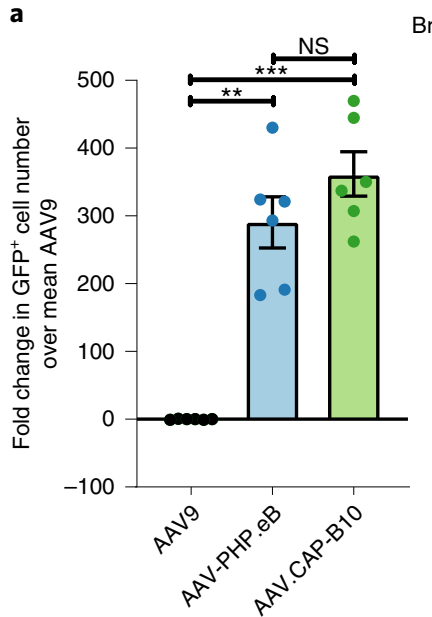

Brain
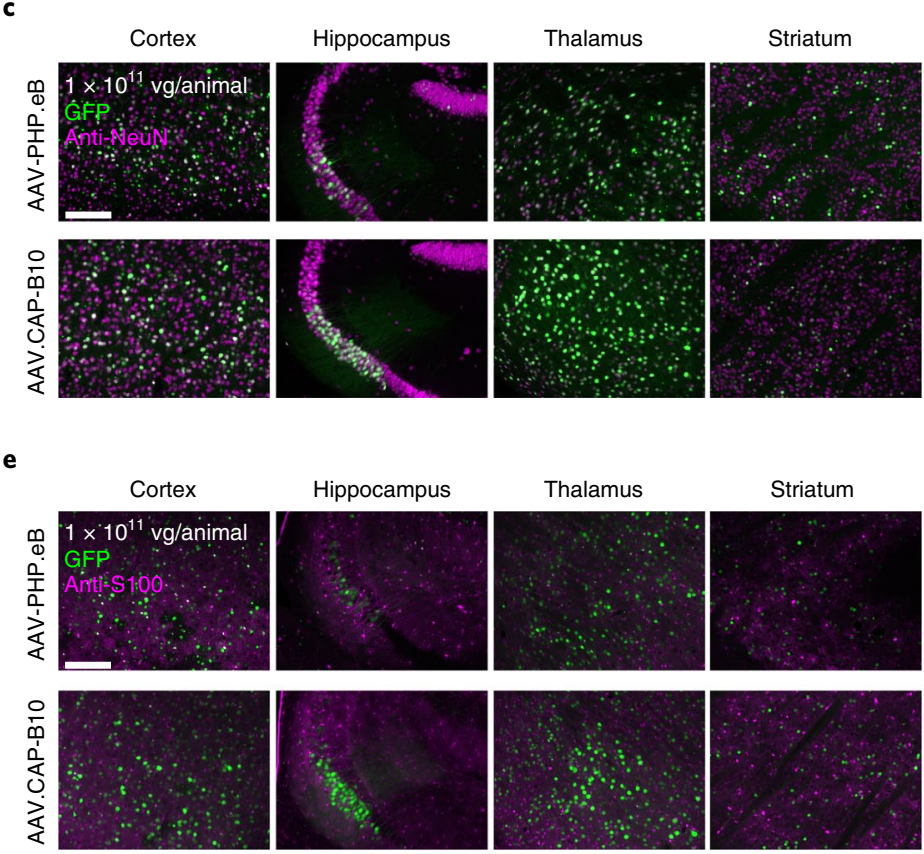

g

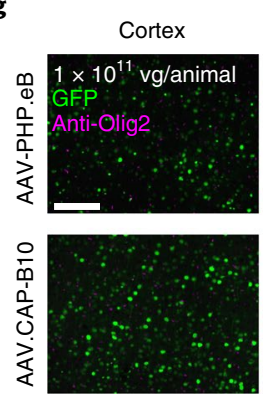

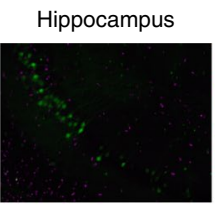

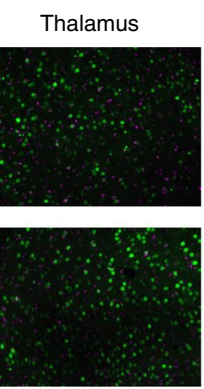

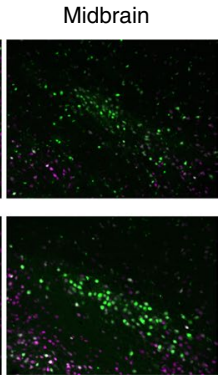
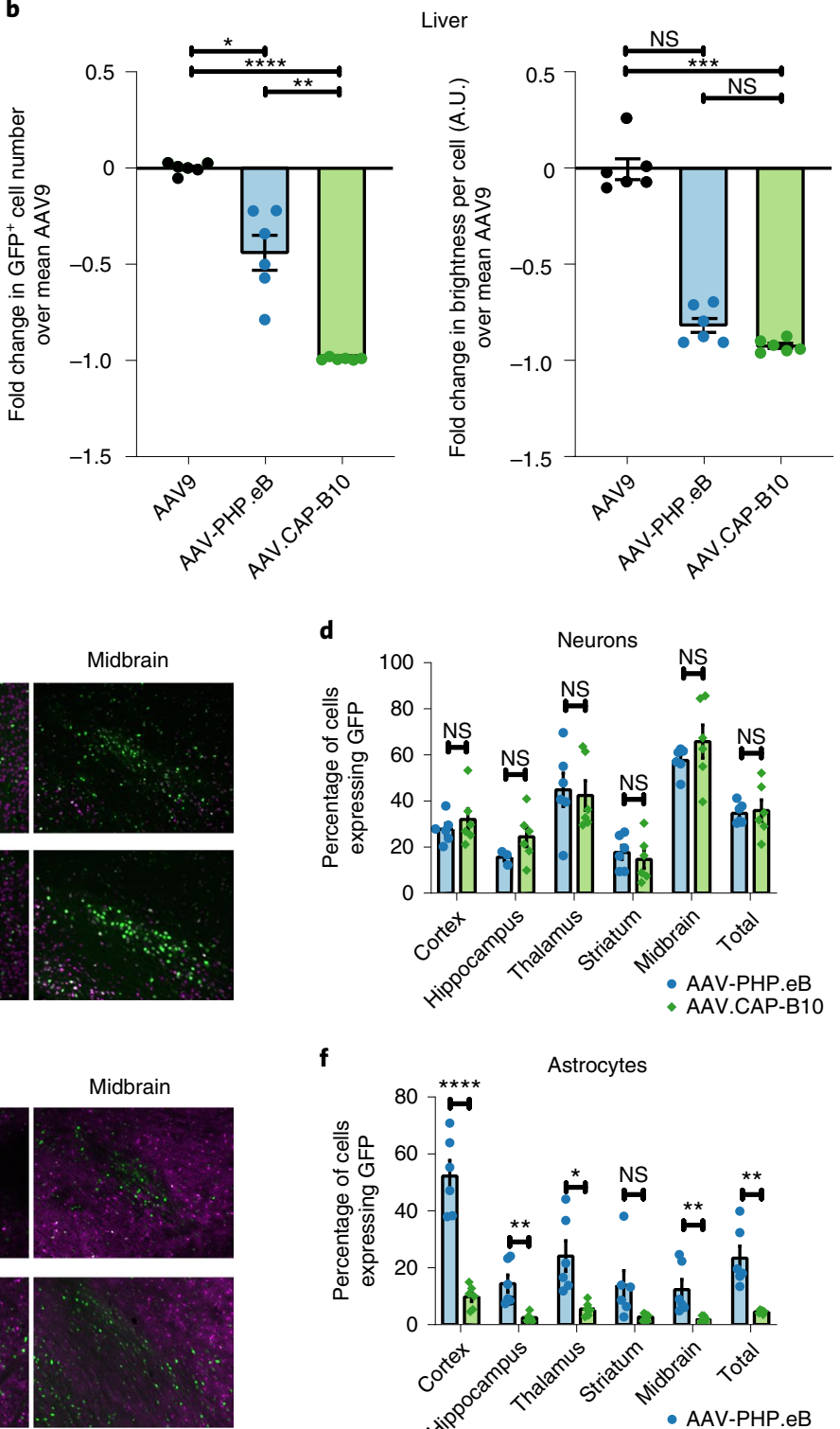

d

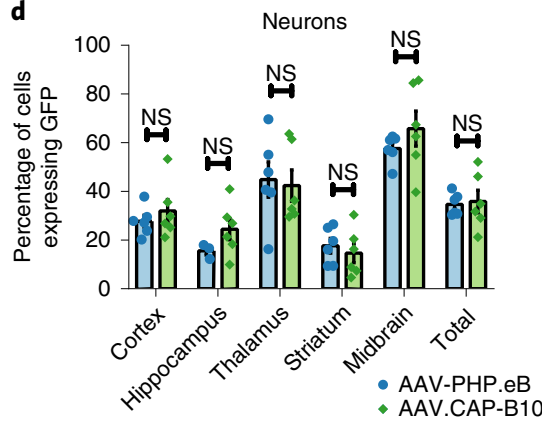

f
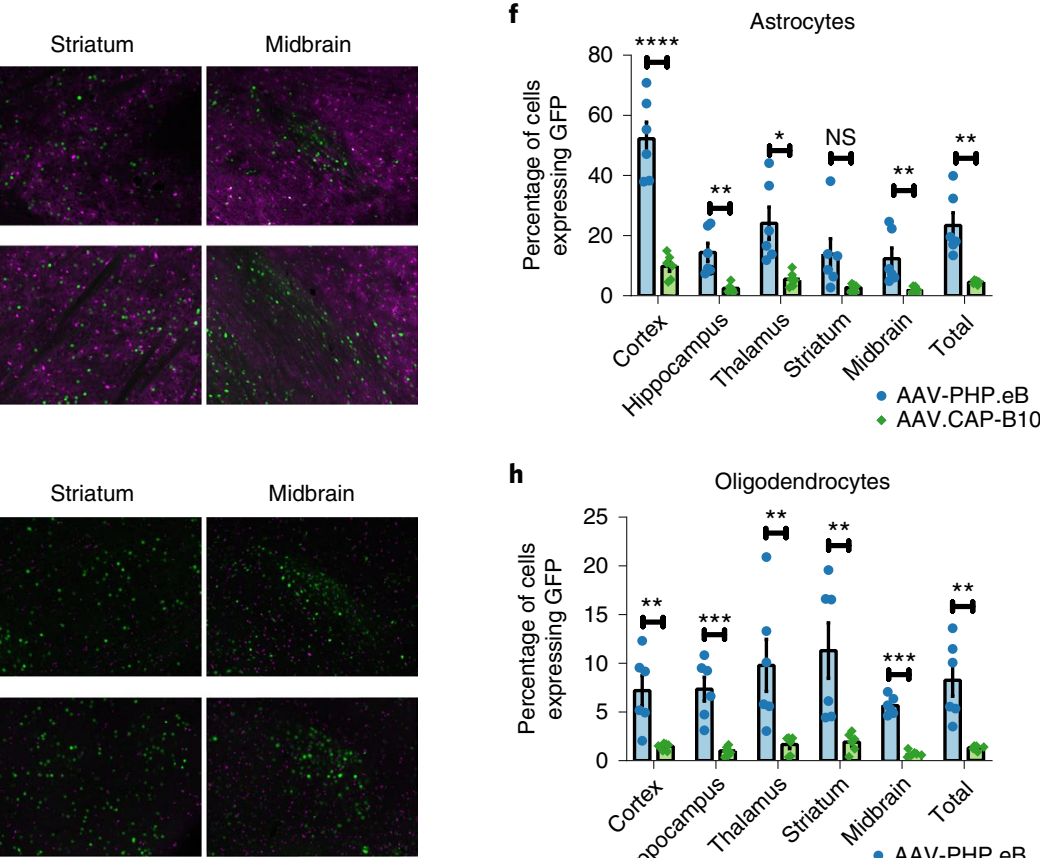

h

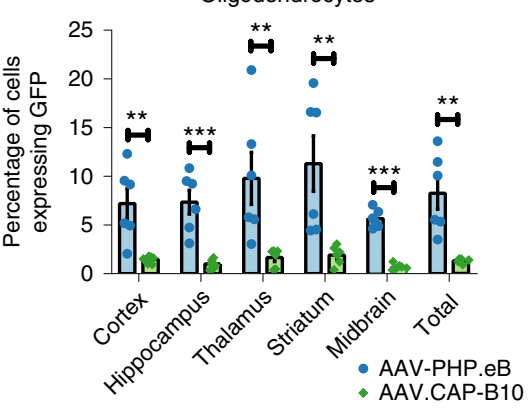




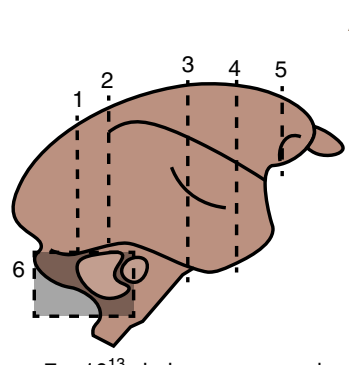

$7 \times 10^{13}$ viral genomes per $\mathrm{kg}$

b

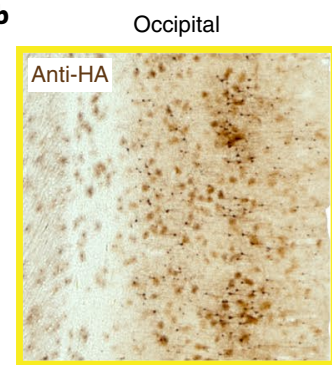

Occipital cortex

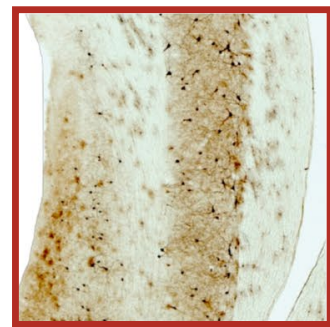

Anti-HA
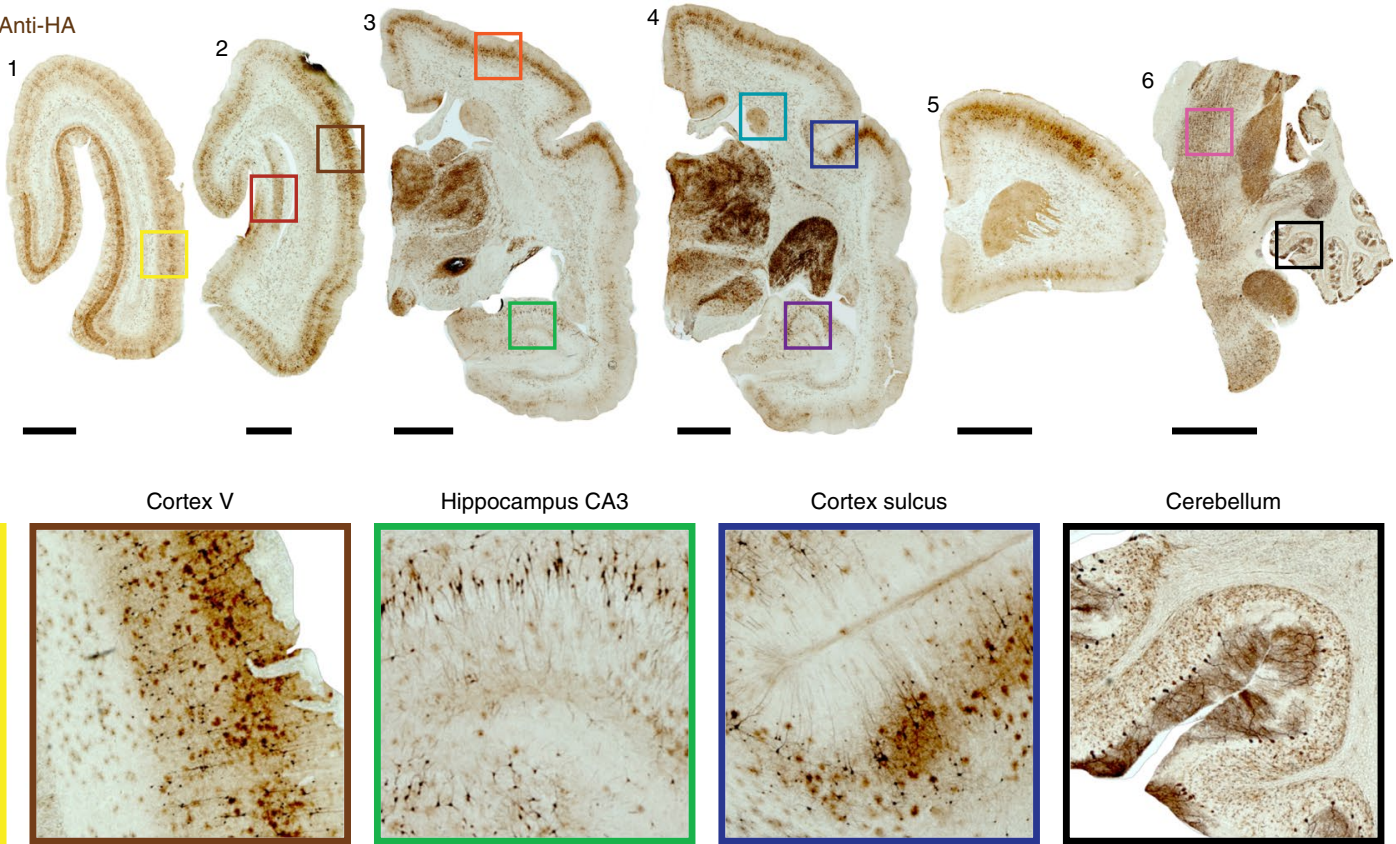

Caudate nucleus
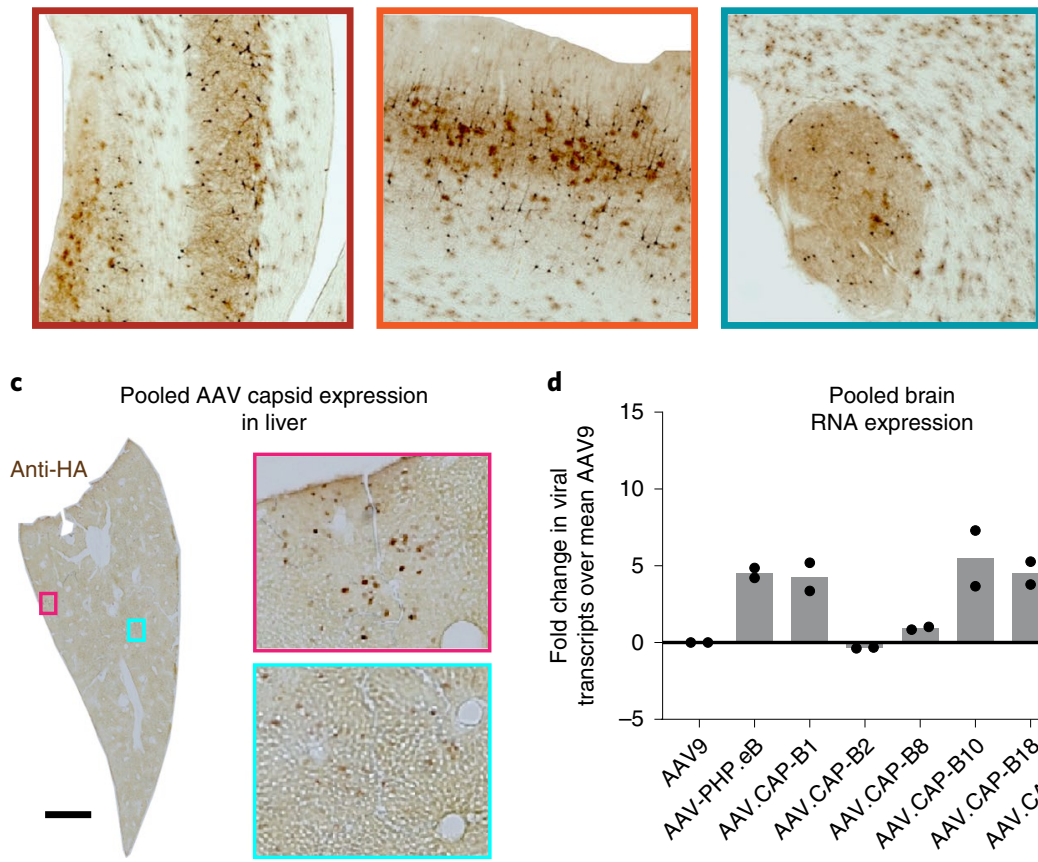

d

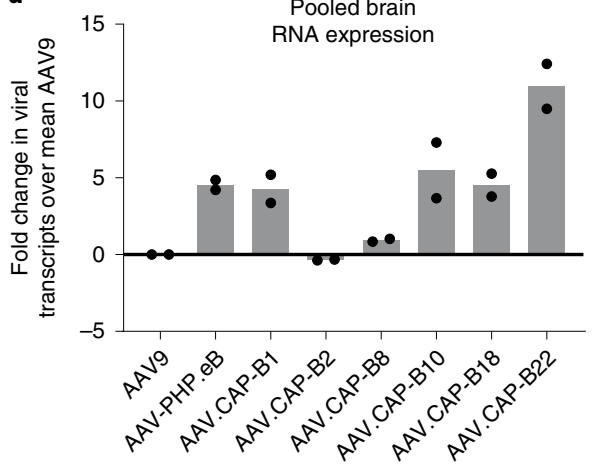

Cortex sulcus

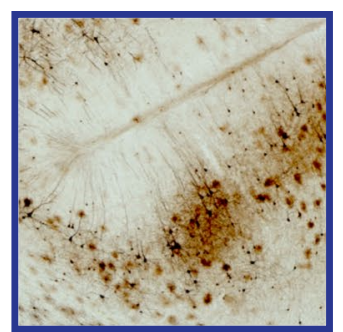

Hippocampus CA3

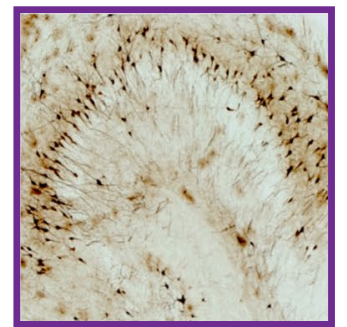

e

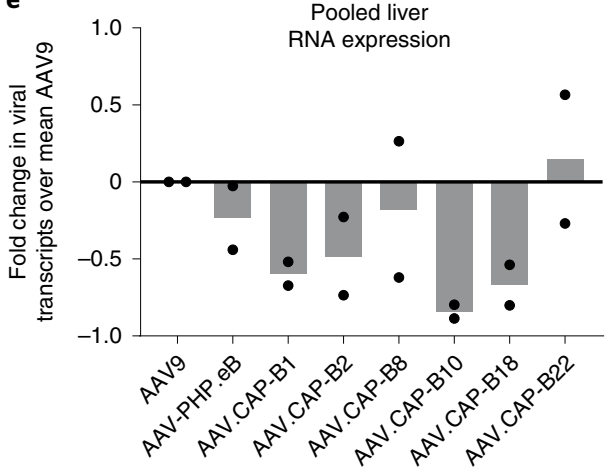

Fig. 3 | Characterization of pooled capsid transgene expression in NHPs. a, AAV.CAP-B1, AAV.CAP-B2, AAV.CAP-B8, AAV.CAP-B10, AAV.CAP-B18 and AAV.CAP-B22, along with AAV9 and AAV-PHP.eB as controls, packaging a human FXN fused to an HA tag under the control of the ubiquitous CAG promoter, were pooled and intravenously injected into two adult marmosets at doses of $1.2 \times 10^{14}(\mathrm{MPV} 1)$ and $7 \times 10^{13}(\mathrm{MPV} 2)$ viral genomes per $\mathrm{kg}$ total. Six sections distributed throughout the brain of one marmoset (MPV2) showed robust expression after immunostaining for the HA tag (Cell Signaling Technologies, C29F). Scale bars, $2 \mathrm{~mm}$. b, Magnified frames from a for a variety of cortical and sub-cortical regions. c, Liver section taken from one marmoset (MPV2) shows minimal expression after immunostaining for the HA tag (Cell Signaling Technologies, C29F). d, NGS quantification within brain tissue of the unique RNA barcode associated with each virus shows a substantial increase for several variants, including a more than 12-fold increase in RNA levels of AAV.CAP-B22 and a fivefold increase for AAV.CAP-B10 compared to AAV9. e, NGS quantification of pooled variant injections, showing relative targeting away from the liver for the different variants. AAV.CAP-B22 contributes similar RNA levels as AAV9, but AAV.CAP-B10 contributes more than fivefold less. $n=2$ marmosets per group. Scale bars, $2 \mathrm{~mm}$.

administration. Initially, we generated a library of AAV capsid sequences, theoretically containing 1.28 billion 7-mer AA substitutions, and produced the corresponding viruses in HEK293 cells that package a replication-incompetent version of their own genome with a polyadenylation sequence flanked by Cre-Lox sites (Fig. 1b). This viral library is then injected into transgenic animals expressing 
Cre recombinase in specific cell populations. Variants that successfully transduce $\mathrm{Cre}^{+}$cells have their genome flipped, and the corresponding sequences are recovered. To exert simultaneous positive and negative selective pressure, we performed parallel selections of this library in multiple transgenic lines expressing Cre recombinase in different cell populations. The differential expression of Cre in the transgenic lines allows for shifts in the tropism of each viral variant to be compared between tissue types, enabling the recovery of unique sequences with a desired transduction profile after only two rounds of selection (for example, strong CNS and weak liver transduction).

We injected viral libraries into four separate Cre transgenic mouse lines to select viral variants specific to the CNS: Tek-Cre for endothelial cells throughout the body; hSyn1-Cre for neurons of the CNS and peripheral nervous system; GFAP-Cre for astrocytes; and TH-Cre for midbrain cells. Using the described Cre-dependent method, we recovered viral DNA from all tissues across each mouse line and identified 434,770 capsid variants capable of tissue transduction through NGS. Afterwards, we ranked variant sequences from each tissue by their enrichment score, defined as the relative abundance of the sequence found within the specific tissue over the relative abundance of that sequence within the injected viral library. After ranking by enrichment, we selected the top $10-20 \%$ of variants with desired profiles from each tissue for the next round of selection.

Capsid engineering refines expression patterns in mice. We used the enriched sequences from the first round of selection to synthesize a library for the second round of selection. The second-round library contained codon sequences of 82,710 unique variants and duplicate, codon-modified versions of each variant as a replicate. Using the same selection methods and transgenic lines as round 1, we determined the enrichment of each variant across tissues and transgenic lines. The second round of selection reduced the number of enriched variants by two orders of magnitude, and we identified 39,034 sequences that result in CNS enrichment and decreased targeting of the liver. Using these sequences, we differentiated a subset of AAs in each position that contributed to this tropism profile (Extended Data Fig. 1). We ranked sequences by their specificity to CNS tissues and compared against the enrichment in peripheral tissues across the different transgenic lines, selecting 22 sequences with diverse sequence identity (Fig. 1c) for further testing in vivo.

To validate the enrichment profile obtained from sequencing, we individually screened each of these 22 capsid variants for their tissue tropism after systemic injection in mice. We produced capsid variants, packaging a mNeonGreen fluorescent reporter under regulation of the ubiquitous CAG promoter, and administered each variant to wild-type (WT) mice by intravenous injection at a dose of $5 \times 10^{11}$ viral genomes per animal. After 2 weeks of expression, we imaged brains and livers and quantified fluorescence to assess $\mathrm{mNeonGreen} \mathrm{expression.} \mathrm{Six} \mathrm{variants} \mathrm{dis-}$ played similar or higher fluorescence in the brain to AAV-PHP.eB as well as reduced liver expression in mice (Fig. 1d): AAV.CAP-B1, AAV.CAP-B2, AAV.CAP-B8, AAV.CAP-B10, AAV.CAP-B18 and AAV.CAP-B22. Owing to the negligible liver expression of AAV. CAP-B10, we characterized this variant further in mice, whereas we selected all six variants for pooled testing in marmosets.

AAV.CAP-B10 yields CNS-specific transgene expression in mice. To characterize the performance of AAV.CAP-B10 in comparison to AAV9 and AAV-PHP.eB, we packaged a nuclear-localized enhanced green fluorescent protein (EGFP) under regulation of a ubiquitously expressed CAG promoter, enabling quantification across cell types throughout the body. The variants were injected into mice at a dose of $1 \times 10^{11}$ viral genomes per animal, and tissue was collected after 3 weeks of expression. Detailed cell counts of overall EGFP expression throughout the body showed that expression after delivery with AAV.CAP-B10 is highly specific to the CNS. In the brain, delivery with AAV.CAP-B10 yielded a similar number of EGFP-expressing cells with a similar level of expression per cell to AAV-PHP.eB, whereas both AAV.CAP-B10 and AAV-PHP.eB had many more cells expressing EGFP than AAV9 (Fig. 2a). In the spinal cord, $40 \%$ fewer cells displayed EGFP expression after delivery with AAV.CAP-B10 than with AAV-PHP.eB but approximately 16 -fold more than AAV9 (Extended Data Fig. 2). Conversely, the number of cells expressing EGFP after delivery with AAV.CAP-B10 was significantly reduced in the liver compared to both AAV-PHP.eB ( 50-fold) and AAV9 (>100-fold). EGFP expression after delivery with AAV.CAP-B10, but not AAV-PHP.eB, was significantly dimmer per cell than AAV9 in the liver ( $\sim$ tenfold) (Fig. 2b). This trend was maintained in the other peripheral organs, with significantly fewer cells displaying EGFP expression after delivery with AAV.CAP-B10 than AAV9 (Extended Data Fig. 2).

AAV.CAP-B10 brain transgene expression is neuronal specific. To further characterize transgene expression in the CNS after delivery with AAV.CAP-B10 compared to AAV-PHP.eB, we co-stained for neurons $(\alpha \mathrm{NeuN})$, astrocytes $(\alpha \mathrm{S} 100)$, oligodendrocytes ( $\alpha \mathrm{Olig} 2)$ and Purkinje cells ( $\alpha$ Calbindin) and quantified the efficiency of EGFP expression after delivery with each capsid in each cell type across various brain regions (Fig. 2c-h and Extended Data Fig. 3). Whereas neurons displayed EGFP expression with similar efficiencies after delivery by AAV.CAP-B10 or AAV-PHP.eB across brain regions (Fig. 2c,d), roughly four- to fivefold fewer astrocytes and oligodendrocytes display EGFP expression after delivery by AAV. CAP-B10 compared to AAV-PHP.eB (Fig. 2e-h). This indicates that

Fig. 4 | Characterization of single-variant expression after delivery with each of AAV9, AAV-PHP.eB, AAV.CAP-B10 and AAV.CAP-B22 in marmosets. Human FXN fused to an HA tag is packaged in each variant under control of the ubiquitous CAG promoter. Marmosets were injected at a dose of $7 \times 10^{13}$ viral genomes per kg (see also Extended Data Figs. 5 and 6). a, Cortical expression is compared for AAV9, AAV-PHP.eB, AAV.CAP-B10 and AAV. CAP-B22 by immunostaining for the HA tag (Roche, 3F10) on the FXN transgene in conjunction with NeuN (Abcam, 177487). A qualitative increase in transgene expression efficiency for AAV.CAP-B10 and AAV.CAP-B22 is observed in comparison to AAV9 and AAV-PHP.eB. Displayed sections are taken from a similar plane and cortical region. Scale bar, $200 \mu \mathrm{m}$. b. Similar regions across all layers of the marmoset cortex are quantified, where AAV.CAP-B10 shows fourfold increase in HA-positive neurons over AAV9 $(P=0.0299)$ and AAV-PHP.eB $(P=0.0295)$, respectively. Differences among other groups are not significant. $n=2$ for AAV9, $n=3$ for AAV-PHP.eB, $n=4$ for AAV.CAP-B10 and $n=4$ for AAV.CAP-B22, mean \pm s.e. Significance is determined by two-tailed Welch's $t$-test. c, Liver expression is compared for AAV9, AAV-PHP.eB, AAV.CAP-B10 and AAV.CAP-B22 by immunostaining for the HA tag (Roche, 3F10) on the FXN transgene in conjunction with DAPI. Scale bar, $200 \mu \mathrm{m}$. d, In the liver, we quantified the fraction of HA-positive cells out of the total nuclei stained, where AAV.CAP-B10 had expression in 17-fold fewer cells than AAV9. No significant differences were observed among AAV-PHP.eB, AAV.CAP-B10 or AAV.CAP-B22. $n=1$ for AAV9, $n=3$ for AAV-PHP.eB, $n=2$ for AAV.CAP-B10 and $n=3$ for AAV.CAP-B22, mean \pm s.e. e, Five sections distributed throughout the brain, spinal cord and DRG of the marmoset show robust expression after immunostaining for the HA tag (Roche, 3F10). Scale bar, $5 \mathrm{~mm}$. f, Magnified frames from e display expression across cortical, sub-cortical, cerebellar, spinal column and DRG regions. Scale bar, $500 \mu \mathrm{m}$. 
the AAV.CAP-B10 mutation confers a bias for neurons over other cell types, an interesting deviation from AAV9, which mostly targets astrocytes in the brain ${ }^{43,44}$. A noteworthy indication from the NGS data for AAV.CAP-B10 was this variant's decreased presence in the cerebellum, which was also evidenced by a decrease in the overall cerebellar fluorescence in the initial characterization (Fig. 1d). When comparing the cerebellar expression after delivery with AAV. CAP-B10 to that of AAV-PHP.eB, we found a significant, roughly fourfold decrease in the number of Purkinje cells displaying EGFP expression (Extended Data Fig. 4).
Engineered variants maintain robust tropism in marmosets. Of primary concern for the therapeutic applicability of variants engineered in rodents is how well their transgene expression profile translate to NHPs. Therefore, we sought to characterize the marmoset CNS transgene expression profile of a subset of the variants validated in mice, along with AAV9 and AAV-PHP.eB as controls. We produced a pool of eight viruses-AAV9, AAV-PHP. eB, AAV.CAP-B1, AAV.CAP-B2, AAV.CAP-B8, AAV.CAP-B10, AAV.CAP-B18 and AAV.CAP-B22-each packaging an HA-tagged frataxin $(\mathrm{FXN})$ with a unique molecular barcode under control of

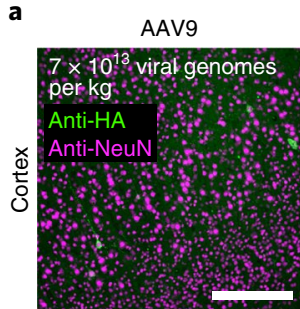

AAV-PHP.eB
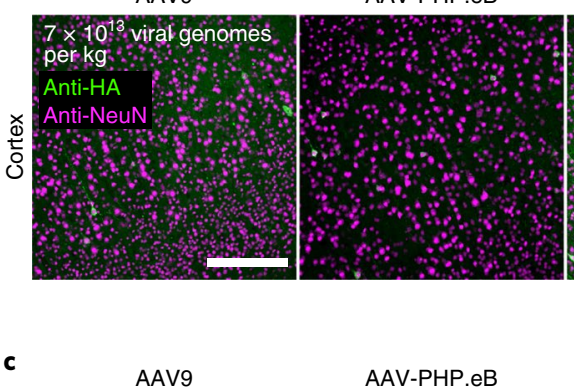

AAV9

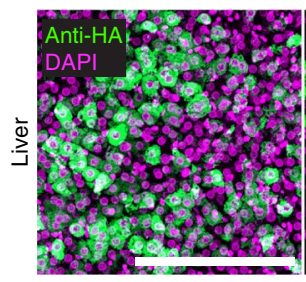

AAV-PHP.eB

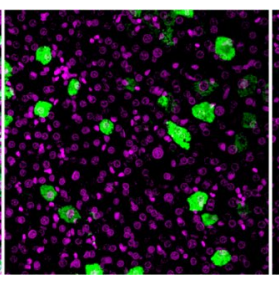

AAV.CAP-B10

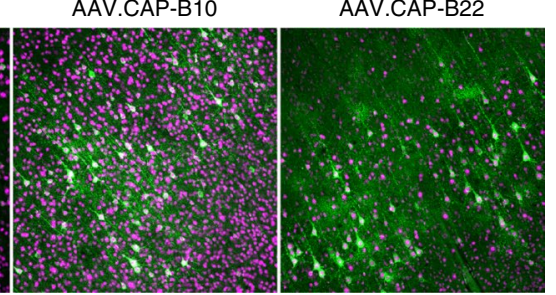

AAV.CAP-B10

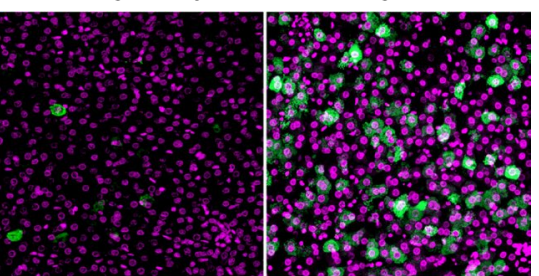

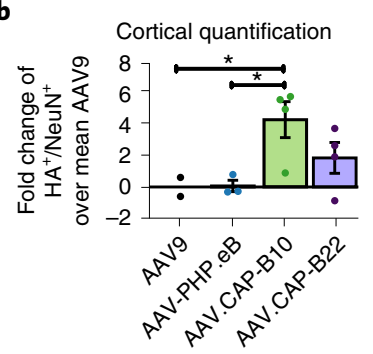

d

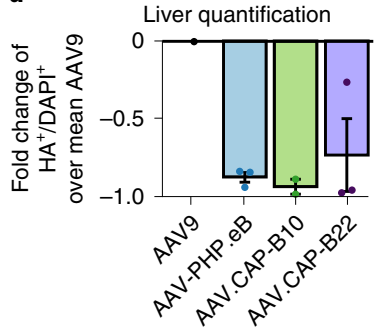

e

AAV.CAP-B10 nervous system tissue expression profile
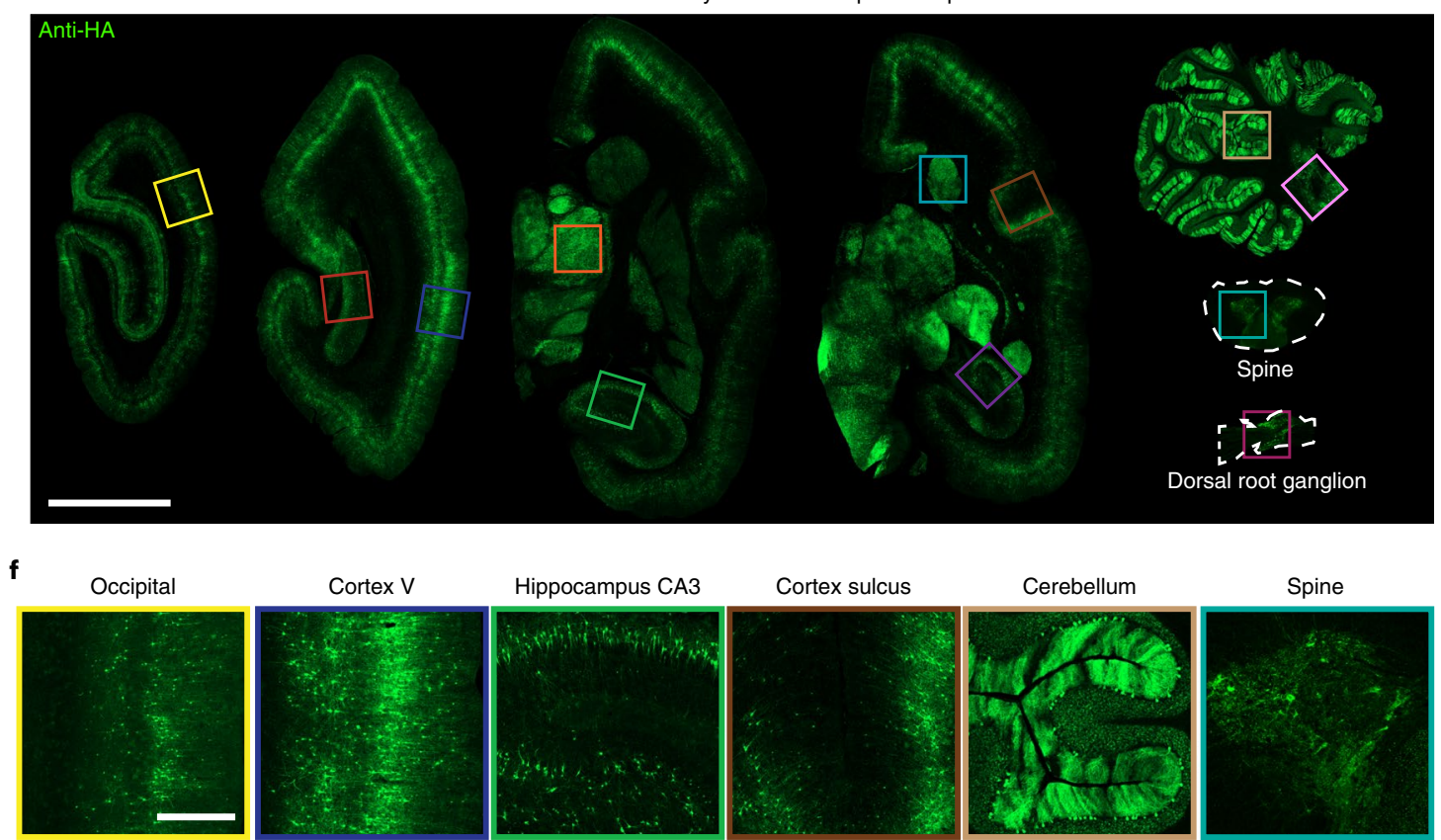

Cortex V

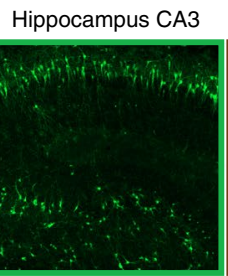

Cortex sulcus

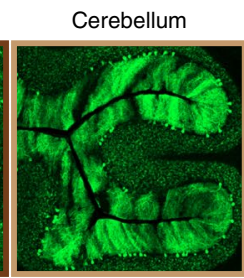

Spine
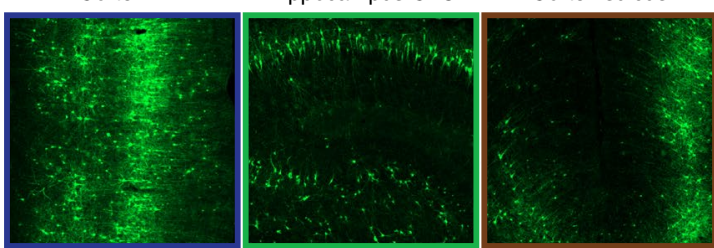

Occipital cortex

Thalamus

Striatum

Hippocampus CA3

Brain stem
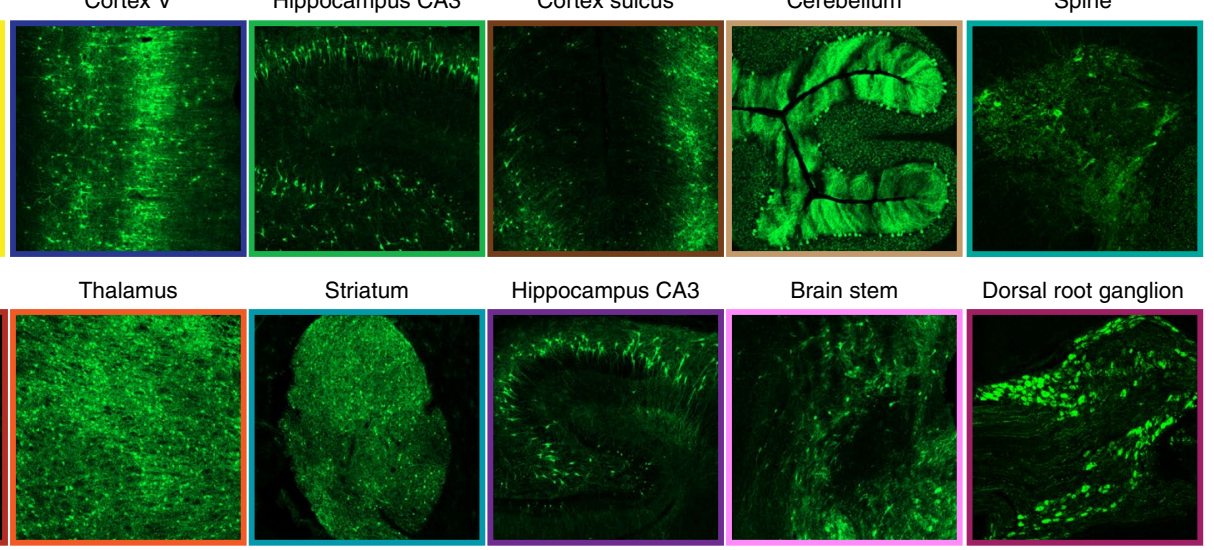
the ubiquitous CAG promoter. We opted to use FXN because it is an endogenous protein expressed throughout the body, and previous efforts to characterize NHP transgene expression after delivery with naturally occurring and engineered serotypes have resulted in deleterious effects for the host, potentially due to the packaging of exogenous transgenes such as GFP ${ }^{30,45,46}$. We also included a separate 12-base RNA barcode into each genome to differentiate the contribution of each virus from the rest after using NGS. The eight viruses were pooled at equal ratios and intravenously injected into two adult marmosets at doses of $1.2 \times 10^{14}$ (marmoset pool virus 1 (MPV1)) and $7 \times 10^{13}$ (MPV2) viral genomes per $\mathrm{kg}$ (Extended Data Table 1). After 6 weeks of expression, during which no adverse health effects were observed, the brains and livers were recovered, and sections were taken for RNA sequencing and immunohistochemistry.

Staining for the HA tag revealed that robust and broad expression was achieved in the adult marmoset brain, with strong expression of the viral pool observed in cortical, subcortical and cerebellar layers throughout (Fig. 3a,b). In the liver, moderate FXN expression was observed after staining for the HA tag (Fig. 3c). From multiple slices per animal distributed throughout the brain and liver, we extracted RNA, performed NGS and quantified the relative expression levels of each of the barcoded viruses. AAV.CAP-B10 recapitulated the trends observed in mice (albeit at lower levels), with a sixfold increase in RNA in the brain and a fivefold decrease in RNA in the liver compared to AAV9 (Fig. 3d,e). AAV.CAP-B22 exhibited the largest increase in RNA levels in the brain, with a greater than 12 -fold increase in the brain compared to AAV9, but relatively similar levels in the liver (Fig. 3d,e). Based on their unique expression profiles in the pooled screen, we selected AAV.CAP-B10 and AAV.CAP-B22 for individual characterization of their CNS transgene expression profile in adult marmosets compared to AAV9 and AAV-PHP.eB.

We intravenously injected individual adult marmosets with AAV9, AAV-PHP.eB, AAV.CAP-B10 or AAV.CAP-B22, each packaging an HA-tagged FXN under the control of the ubiquitous $C A G$ promoter, at a dose of $7 \times 10^{13}$ viral genomes per $\mathrm{kg}$ (Extended Data Table 2). The brains were recovered, and sections were taken for sequencing and immunofluorescence between $34 \mathrm{~d}$ and $42 \mathrm{~d}$ after intravenous injection. To characterize the CNS transgene expression after delivery with the four variants, we stained sections for the presence of the HA tag. In stained sections, both AAV.CAP-B10 and AAV.CAP-B22 conditions displayed a marked increase in FXN expression efficiency within cortical regions in comparison to AAV9 and AAV-PHP.eB (Fig. 4a). Each of AAV-PHP.eB, AAV. CAP-B10 and AAV.CAP-B22 displayed reduction in liver FXN expression relative to AAV9 (Fig. 4C).

After delivery with AAV.CAP-B22, FXN expression was observed across broad cell types in the marmoset brain. Qualitatively, many more astrocytes (measured by co-staining with S100 $\beta$ ) displayed FXN expression compared to delivery with AAV9 or AAV.CAP-B10 (Extended Data Fig. 5). The FXN expression levels of AAV.CAP-B22 had high variability among animals in both the brain and liver, and, therefore, we observed no significant difference in the neuronal FXN expression after delivery with AAV.CAP-B22 relative to AAV9 or AAV-PHP.eB.

TheneuronalspecificityofAAV.CAP-B10inmicewasrecapitulated in marmoset brains, where HA-positive cells were highly correlated with NeuN staining across brain regions. In the marmoset cortex, AAV.CAP-B10 displays a statistically significant $\sim$ fourfold increase in HA-positive neurons over AAV9 and AAV-PHP.eB (Fig. 4b). Notably, this increased protein expression in cortical neurons after delivery with AAV.CAP-B10 occurs despite no significant difference between the bulk viral genome and transcript measurements of AAV.CAP-B10 and AAV9 or AAV-PHP.eB (Extended Data Fig. 6b), further highlighting tropism differences between AAV9 and AAV. CAP-B10. The decreased liver targeting of AAV.CAP-B10 in mice was also recapitulated in the marmoset, where there were $\sim 17$-fold fewer cells expressing the transgene compared to an AAV9 control (Fig. 4d). Viral genome and transcript measurements corroborate the protein expression data in the liver (Extended Data Fig. 6a). We observed broad and robust transgene expression after delivery with AAV.CAP-B10 across cortical, sub-cortical and cerebellar regions as well as in the spinal column and the dorsal root ganglia (DRG) (Fig. 4e,f).

\section{Discussion}

The power of directed evolution and AAV engineering to confer novel tropisms and tissue specificity has broadened potential research applications and enabled new therapeutic approaches in the $\mathrm{CNS}^{47-52}$. Our results show that introducing diversity at multiple locations on the capsid surface into native or previously engineered variants can provide useful features, such as increased transgene expression efficiency and tissue or neuronal specificity in WT mice and marmosets. This finding has broad implications for the field of AAV engineering, and the variants discovered using the M-CREATE method ${ }^{22,26,33}$ have the potential to provide targeted gene delivery in WT animals.

Novel variants from this library achieved overall similar levels of transgene expression throughout the mouse brain as the previously engineered parent AAV (AAV-PHP.eB) but with striking deviations in cell type specificity and targeting or, notably, targeting away from other organs. Across the two engineering efforts, iterative modifications made to adjacent loops first conferred a new phenotype-the ability to cross the blood-brain barrier efficiently-and then refined the phenotype away from other organs or toward specific cell types. Most notably from this dataset, AAV.CAP-B10 is targeted away from the entire periphery and exhibits specificity for neurons over other cell types in the CNS. Of the 82,710 variants that comprised our second round of selection, roughly 39,000 exhibited positive enrichment in the brain and negative enrichment in the liver. Of those, the variants tested in vivo in mice showed expression patterns that correlated with their NGS enrichments and rankings. Furthermore, the presence of additional sequences that were positively enriched in the brain and negatively enriched in peripheral organs indicates the richness of this dataset and engineering location, with many more potentially interesting variants to be identified and characterized. Together, these results indicate that future engineering efforts can also take a stepwise approach toward attaining specificity for certain targets, with each engineering round refining and enhancing novel tropisms identified in the previous round. Notably, this implies that AAV.CAP-B10 might be further refined in its specificity to neuronal sub-classes ${ }^{53,54}$ in the CNS or away from the DRG, which has been associated with toxicity in NHP studies ${ }^{55}$.

The therapeutic use of engineered AAVs for treating disease has increased exponentially in recent years, including the use of systemically administered AAVs for CNS gene therapies. The brain is a prime target for gene therapy of a wide array of diseases, such as Huntington's disease, Parkinson's disease and Friedreich's ataxia ${ }^{56-58}$, and, by developing gene therapy vectors for systemic administration, we can enable the wide coverage and non-invasive delivery conducive to effective treatment of these diseases. To minimize side effects typically associated with systemic delivery, gene therapy vectors should be engineered for specificity toward the therapeutic target of interest in conjunction with decreased off-target expression. Basic research in NHP brains, compared to rodent research, has been hampered by few options for genetic access. Gene delivery vectors could bridge this gap and enable eventual use in gene therapy applications. However, the translation of engineered variants from mice to NHPs has been challenging. Two generations of bloodbrain-barrier-crossing engineered AAV variants (AAV-PHP.B and AAV-PHP.eB) have failed ${ }^{34}$ to increase transgene expression in the brain after systemic injection in NHPs. Therefore, variants 
engineered in this study, including AAV.CAP-B10 and AAV. CAP-B22, represent notable progress toward evolving variants that are capable of efficiently, specifically and safely delivering gene therapies to the CNS in NHPs and, ultimately, in humans. Robust transgene expression in the CNS, decreased liver targeting and neuronal bias of AAV.CAP-B10 observed after two rounds of selection in mice were recapitulated in marmosets, indicating that M-CREATE in rodents can be effectively used as a screening platform before direct screening or validation in NHPs, thus greatly increasing the throughput of the engineering process. Together, these results constitute an important step forward toward achieving the goal of engineered AAV vectors that can be used to broadly deliver gene therapies to the CNS in humans.

\section{Online content}

Any methods, additional references, Nature Research reporting summaries, source data, extended data, supplementary information, acknowledgements, peer review information; details of author contributions and competing interests; and statements of data and code availability are available at https://doi.org/10.1038/ s41593-021-00969-4.

Received: 16 June 2020; Accepted: 22 October 2021;

Published online: 9 December 2021

\section{References}

1. Naso, M. F., Tomkowicz, B., Perry, W. L. \& Strohl, W. R. Adeno-associated virus (AAV) as a vector for gene therapy. BioDrugs 31, 317-334 (2017).

2. Gaudet, D., Méthot, J. \& Kastelein, J. Gene therapy for lipoprotein lipase deficiency. Curr. Opin. Lipidol. 23, 310-320 (2012).

3. Al-Zaidy, S. A. \& Mendell, J. R. From clinical trials to clinical practice: practical considerations for gene replacement therapy in SMA type 1. Pediatr. Neurol. 100, 3-11 (2019).

4. Ameri, H. Prospect of retinal gene therapy following commercialization of voretigene neparvovec-rzyl for retinal dystrophy mediated by RPE65 mutation. J. Curr. Ophthalmol. 30, 1-2 (2018).

5. Evens, H., Chuah, M. K. \& VandenDriessche, T. Haemophilia gene therapy: from trailblazer to gamechanger. Haemophilia 24, 50-59 (2018).

6. Ginn, S. L., Amaya, A. K., Alexander, I. E., Edelstein, M. \& Abedi, M. R. Gene therapy clinical trials worldwide to 2017: an update. J. Gene Med. 20, e3015 (2018).

7. Srivastava, A. In vivo tissue-tropism of adeno-associated viral vectors. Curr Opin. Virol. 21, 75-80 (2016)

8. Zincarelli, C., Soltys, S., Rengo, G. \& Rabinowitz, J. E. Analysis of AAV serotypes 1-9 mediated gene expression and tropism in mice after systemic injection. Mol. Ther. 16, 1073-1080 (2008).

9. Gray, S. J., Woodard, K. T. \& Samulski, R. J. Viral vectors and delivery strategies for CNS gene therapy. Ther. Deliv. 1, 517-534 (2010).

10. Golebiowski, D. et al. Direct intracranial injection of AAVrh8 encoding monkey $\beta$-N-acetylhexosaminidase causes neurotoxicity in the primate brain. Hum. Gene Ther. 28, 510-522 (2017)

11. Hocquemiller, M., Giersch, L., Audrain, M., Parker, S. \& Cartier, N. Adeno-associated virus-based gene therapy for CNS diseases. Hum. Gene Ther. 27, 478-496 (2016).

12. Kubes, P. \& Jenne, C. Immune responses in the liver. Annu. Rev. Immunol.

36, 247-277 (2018).

13. Gao, B. Basic liver immunology. Cell. Mol. Immunol. 13, 265-266 (2016).

14. Ronzitti, G., Gross, D.-A. \& Mingozzi, F. Human immune responses to adeno-associated virus (AAV) vectors. Front. Immunol. 11, 670 (2020).

15. Verdera, H. C., Kuranda, K. \& Mingozzi, F. AAV vector immunogenicity in humans: a long journey to successful gene transfer. Mol. Ther. 28, 723-746 (2020).

16. Gray, S. J. et al. Optimizing promoters for recombinant adeno-associated virus-mediated gene expression in the peripheral and central nervous system using self-complementary vectors. Hum. Gene Ther. 22, 1143-1153 (2011)

17. de Leeuw, C. N. et al. Targeted CNS delivery using human MiniPromoters and demonstrated compatibility with adeno-associated viral vectors. Mol. Ther. Methods Clin. Dev. 1, 5 (2014).

18. Allen, W. E. et al. Global representations of goal-directed behavior in distinct cell types of mouse neocortex. Neuron 94, 891-907 (2017).

19. Shima, Y. et al. A mammalian enhancer trap resource for discovering and manipulating neuronal cell types. eLife 5, e13503 (2016).

20. Graybuck, L. T. et al. Enhancer viruses for combinatorial cell-subclass-specific labeling. Neuron 109, 1449-1464 (2021).
21. Mich, J. K. et al. Functional enhancer elements drive subclass-selective expression from mouse to primate neocortex. Cell Rep. 34, 108754 (2021).

22. Chan, K. Y. et al. Engineered AAVs for efficient noninvasive gene delivery to the central and peripheral nervous systems. Nat. Neurosci. 20, 1172-1179 (2017).

23. Vormstein-Schneider, D. et al. Viral manipulation of functionally distinct interneurons in mice, non-human primates and humans. Nat. Neurosci. 23, 1629-1636 (2020).

24. Keaveney, M. K. et al. A microRNA-based gene-targeting tool for virally labeling interneurons in the rodent cortex. Cell Rep. 24, 294-303 (2018).

25. Hordeaux, J. et al. MicroRNA-mediated inhibition of transgene expression reduces dorsal root ganglion toxicity by AAV vectors in primates. Sci. Transl. Med. 12, eaba9188 (2020).

26. Deverman, B. E. et al. Cre-dependent selection yields AAV variants for widespread gene transfer to the adult brain. Nat. Biotechnol. 34, 204-209 (2016)

27. Zelikowsky, M. et al. The neuropeptide Tac2 controls a distributed brain state induced by chronic social isolation stress. Cell 173, 1265-1279 (2018)

28. Zeng, J. et al. TRIM9-mediated resolution of neuroinflammation confers neuroprotection upon ischemic stroke in mice. Cell Rep. 27, 549-560 (2019).

29. Graybuck, L. T. et al. Enhancer viruses for combinatorial cell-subclass-specific labeling. Neuron 109, 1449-1464.e13 (2021).

30. Gao, G. et al. Adeno-associated virus-mediated gene transfer to nonhuman primate liver can elicit destructive transgene-specific T cell responses. Hum. Gene Ther. 20, 930-942 (2009).

31. Mingozzi, F. \& High, K. A. Immune responses to AAV vectors: overcoming barriers to successful gene therapy. Blood 122, 23-36 (2013).

32. Calcedo, R., Chichester, J. A. \& Wilson, J. M. Assessment of humoral, innate, and T-cell immune responses to adeno-associated virus vectors. Hum. Gene Ther. Methods 29, 86-95 (2018).

33. Kumar, S. et al. Multiplexed Cre-dependent selection yields systemic AAVs for targeting distinct brain cell types. Nat. Methods 17, 541-550 (2020).

34. Matsuzaki, Y. et al. Intravenous administration of the adeno-associated virus-PHP.B capsid fails to upregulate transduction efficiency in the marmoset brain. Neurosci. Lett. 665, 182-188 (2018).

35. Hordeaux, J. et al. The neurotropic properties of AAV-PHP.B are limited to C57BL/6J mice. Mol. Ther. 26, 664-668 (2018).

36. Kern, A. et al. Identification of a heparin-binding motif on adeno-associated virus type 2 capsids. J. Virol. 77, 11072-11081 (2003).

37. Girod, A. et al. Genetic capsid modifications allow efficient re-targeting of adeno-associated virus type 2. Nat. Med. 5, 1052-1056 (1999).

38. Michelfelder, S. et al. Peptide ligands incorporated into the threefold spike capsid domain to re-direct gene transduction of AAV8 and AAV9 in vivo. PLoS ONE 6, e23101 (2011).

39. Shen, S., Bryant, K. D., Brown, S. M., Randell, S. H. \& Asokan, A. Terminal $\mathrm{N}$-linked galactose is the primary receptor for adeno-associated virus 9. J. Biol. Chem. 286, 13532-13540 (2011).

40. Summerford, C., Johnson, J. S. \& Samulski, R. J. AAVR: a multi-serotype receptor for AAV. Mol. Ther. 24, 663-666 (2016).

41. Zhang, R. et al. Adeno-associated virus 2 bound to its cellular receptor AAVR. Nat. Microbiol. 4, 675-682 (2019).

42. DiMattia, M. A. et al. Structural insight into the unique properties of adeno-associated virus serotype 9. J. Virol. 86, 6947-6958 (2012).

43. Foust, K. D. et al. Intravascular AAV9 preferentially targets neonatal neurons and adult astrocytes. Nat. Biotechnol. 27, 59-65 (2009).

44. Samaranch, L. et al. Adeno-associated virus serotype 9 transduction in the central nervous system of nonhuman primates. Hum. Gene Ther. 23, 382-389 (2012).

45. Flotte, T. R. \& Büning, H. Severe toxicity in nonhuman primates and piglets with systemic high-dose administration of adeno-associated virus serotype 9-like vectors: putting patients first. Hum. Gene Ther. 29, 283-284 (2018).

46. Unzu, C. et al. Transient and intensive pharmacological immunosuppression fails to improve AAV-based liver gene transfer in non-human primates. J. Transl. Med. 10, 122 (2012).

47. Giannelli, S. G. et al. Cas9/sgRNA selective targeting of the $\mathrm{P} 23 \mathrm{H}$ Rhodopsin mutant allele for treating retinitis pigmentosa by intravitreal AAV9. PHP.B-based delivery. Hum. Mol. Genet. 27, 761-779 (2018).

48. Schubert, R. et al. Virus stamping for targeted single-cell infection in vitro and in vivo. Nat. Biotechnol. 36, 81-88 (2017).

49. Morabito, G. et al. AAV-PHP.B-mediated global-scale expression in the mouse nervous system enables GBA1 gene therapy for wide protection from synucleinopathy. Mol. Ther. 25, 2727-2742 (2017).

50. Hillier, D. et al. Causal evidence for retina-dependent and-independent visual motion computations in mouse cortex. Nat. Neurosci. 20 960-968 (2017).

51. Dayton, R. D., Grames, M. S. \& Klein, R. L. More expansive gene transfer to the rat CNS: AAV PHP.EB vector dose-response and comparison to AAV PHP.B. Gene Ther. 25, 392-400 (2018). 
52. Rincon, M. Y. et al. Widespread transduction of astrocytes and neurons in the mouse central nervous system after systemic delivery of a self-complementary AAV-PHP.B vector. Gene Ther. 25, 83-92 (2018).

53. Yuste, R. et al. A community-based transcriptomics classification and nomenclature of neocortical cell types. Nat. Neurosci. 23, 1456-1468 (2020)

54. Krienen, F. M. et al. Innovations present in the primate interneuron repertoire. Nature 586, 262-269 (2020).

55 . Hinderer, C. et al. Severe toxicity in nonhuman primates and piglets following high-dose intravenous administration of an adeno-associated virus vector expressing human SMN. Hum. Gene Ther. 29 285-298 (2018).
56. Coune, P. G., Schneider, B. L. \& Aebischer, P. Parkinson's disease: gene therapies. Cold Spring Harb. Perspect. Med. 2, a009431 (2012).

57. Piguet, F. et al. Rapid and complete reversal of sensory ataxia by gene therapy in a novel model of Friedreich ataxia. Mol. Ther. 26, 1940-1952 (2018).

58. Combs, B., Kneynsberg, A. \& Kanaan, N. M. Gene therapy models of alzheimer's disease and other dementias. Methods Mol. Biol. 1382, 339-366 (2016)

Publisher's note Springer Nature remains neutral with regard to jurisdictional claims in published maps and institutional affiliations.

(C) The Author(s), under exclusive licence to Springer Nature America, Inc. 2021 


\section{Methods}

Plasmids. The first-round viral DNA library was generated by amplification of a section of the AAV-PHP.eB capsid genome between AA450 and AA599 using NNK degenerate primers (Integrated DNA Technologies) to substitute AA452-AA458 with all possible variations. The resulting library inserts were then introduced into the rAAV- $\Delta$ Cap-in-cis-Lox plasmid via Gibson assembly as previously described ${ }^{26}$ The resulting capsid DNA library, rAAV-Cap-in-cis-Lox, contained a diversity of $\sim 1.28$ billion variants at the AA level. The second-round viral DNA library was generated similarly to the first round, but, instead of NNK degenerate primers at the AA452-AA458 location, a synthesized oligo pool (Twist Bioscience) was used to generate only selected variants. This second-round DNA library contained a diversity of $\sim 82,000$ variants at the AA level.

The AAV2/9 REP-AAP- $\Delta$ Cap plasmid transfected into HEK293T cells for library viral production was modified from the AAV2/9 REP-AAP plasmid previously used ${ }^{26}$ by deletion of the AAs between 450 and 592. This modification prevents production of a WT AAV9 capsid during viral library production after a plausible recombination event between this plasmid co-transfected with rAAV- $\Delta$ Cap-in-cis-Lox containing the library inserts.

Three rAAV genomes were used in this study. The first, pAAV-CAG-mNeonGreen (Addgene, 99134), uses a single-stranded (ss) rAAV genome containing the fluorescent protein $\mathrm{mNeonGreen}$ under control of the ubiquitous CMV- $\beta$-actin-intron- $\alpha$-globin hybrid promoter (CAG). The second, pAAV-CAG-NLS-GFP (Addgene, 104061), uses an ssAAV genome containing the fluorescent protein EGFP flanked by two nuclear localization sites, PKKKRKV, under control of the CAG promoter. The third, pAAV-CAG-FXN-HA, uses an ssAAV genome containing an HA-tagged FXN protein under control of the CAG promoter and harboring a unique 12-bp sequence in the $3^{\prime}$ untranslated region to differentiate different capsids packaging the same construct.

Viral production. rAAVs were generated according to established protocols $\mathrm{s}^{59}$. In brief, HEK293T cells (American Type Culture Collection) were triple transfected using polyethylenimine; virus was collected after $120 \mathrm{~h}$ from both cell lysates and media and purified over iodixanol (OptiPrep, Sigma-Aldrich). The isolated variants investigated in vivo (AAV.CAP-B1, AAV.CAP-B2, AAV.CAP-B8, AAV. CAP-B10, AAV.CAP-B18 and AAV.CAP-B22) have similar production titer to AAV9, with normal titers around $1 \pm 0.7 \times 10^{12}$ viral genomes per $15-\mathrm{cm}$ dish.

A modified protocol was used for transfection and purification of viral libraries. First, to prevent mosaic capsid formation, only $10 \mathrm{ng}$ of rAAV-Cap-in-cis-Lox library DNA was transfected (per 150-mm plate) to decrease the likelihood of multiple library DNAs entering the same cell. Second, virus was collected after $60 \mathrm{~h}$, instead of $120 \mathrm{~h}$, to limit secondary transduction of producer cells. Finally, instead of polyethylene glycol precipitation of the viral particles from the media, as performed in the standard protocol, media were concentrated more than 60 -fold for loading onto iodixanol.

Animals. All rodent procedures were approved by the Institutional Animal Use and Care Committee of the California Institute of Technology. Transgenic animals, expressing Cre under the control of various cell-type-specific promoters, and C57Bl/6J WT mice (000664) were purchased from Jackson Laboratory. Transgenic mice included Syn1-Cre (3966), GFAP-Cre (012886), Tek-Cre (8863) and TH-Cre (008601). Mice were housed under standard conditions between $71^{\circ} \mathrm{F}$ and $75^{\circ} \mathrm{F}$, in $30-70 \%$ humidity and on a light cycle of $13 \mathrm{~h}$ on and $11 \mathrm{~h}$ off. For round 1 and round 2 selections from the viral library, we used one male and one female mouse from each transgenic line (aged 8-12 weeks), as well as a single male C57Bl/6J mouse. For validation of individual viral variants, male C57Bl/6J mice aged 6-8 weeks were used. Intravenous administration of rAAV vectors was performed via injection into the retro-orbital sinus.

Marmoset (C. jacchus) procedures for MPV1 and MPV2 and for marmoset single variant 1-4 (MSV1-4) and MPV11-13 were approved by the Animal Care and Use Committee of the National Institutes of Mental Health (NIMH). MPV1, MPV2, MSV1-4 and MSV11-13 were born and raised in NIMH colonies and housed in family groups under standard conditions of $27^{\circ} \mathrm{C}$ and $50 \%$ humidity. They were fed ad libitum and received enrichment as part of the primate enrichment program for NHPs at the National Institutes of Health (NIH). For AAV infusions, animals were screened for endogenous neutralizing antibodies. None of the animals that were screened showed any detectible blocking reaction at 1:5 dilution of serum (Penn Vector Core, University of Pennsylvania). They were then housed individually for several days and acclimated to a new room before injections. Two animals were used for the pooled injection study, both males, aged 7.6 (MPV1) and 11.5 (MPV2) years (Extended Data Table 1). Five animals were injected with single variants for characterization, but only four were usable (Extended Data Table 2), as one animal (AAV.CAP-B22 injected, 6.9 years, female, $0.475 \mathrm{~kg}$ ) was found dead ( $27 \mathrm{~d}$ after injection), and, at necropsy, the pathology report indicated chronic nephritis unrelated to the virus. The day before infusion, the animals' food was removed. Animals were anesthetized with isoflurane in oxygen; the skin over the femoral vein was shaved and sanitized with an isopropanol scrub; and the virus (Extended Data Tables 1 and 2) was infused over several minutes. Anesthesia was withdrawn, and the animals were monitored until they became active, upon which they were returned to their cages. Activity and behavior were closely monitored over the next $3 \mathrm{~d}$, with daily observations thereafter.

Marmoset procedures for MSV8-10 were approved by the Committee on Animal Care of the Massachusetts Institute of Technology (MIT), and all experiments were performed in accordance with the relevant guidelines and regulations. Marmosets were born and raised in an MIT facility accredited by the Association for Assessment and Accreditation of Laboratory Animal Care. Marmosets were housed in social groups under standard conditions of $23.3 \pm 1.1^{\circ} \mathrm{C}, 50 \% \pm 20 \%$ humidity and a 12 -h light/dark cycle. They were fed ad libitum with standard diet as well as fruits, vegetables and various protein sources. Periodic neutralizing antibody testing of animals in the facility did not reveal significant levels of neutralizing antibodies against AAV9. Each of the animals was injected with single variants for characterization. The day before infusion, the animals' food was removed. Animals were sedated by alfaxalone; the skin over the cephalic vein was shaved and sanitized with an isopropanol scrub; and the virus (Extended Data Table 2) was infused through a 24-gauge catheter over several minutes. After the viral infusion was completed, animals were recovered on a warm water blanket $\left(38^{\circ} \mathrm{C}\right)$ until they regained normal motor functions. Then, animals were returned to their cages and monitored closely for normal behavior over the next $4 \mathrm{~d}$, followed by daily observations thereafter.

Marmoset procedures for MSV5-7 were approved by the Institutional Animal Care and Use Committee of Shenzhen Institute of Advanced Technology (SIAT), Chinese Academy of Sciences. Marmosets were born and raised in SIAT colonies and housed in family groups under standard conditions of $22 \pm 1{ }^{\circ} \mathrm{C}$ and $40-70 \%$ relative humidity. The marmoset breeding and housing facilities are accredited by the Association for Assessment and Accreditation of Laboratory Animal Care. Although animals were not screened for endogenous neutralizing antibodies, the animals were born and raised in the animal facility, and the housing environment for each animal was clean and isolated to prevent bacterial and viral infection. Therefore, the possibility of the animal carrying neutralizing antibodies for AAV virus is low. Before injection, marmosets were separated from family groups, housed two animals per each room for several days and acclimated to a new room before injections. Each of the animals was injected with single variants for characterization, but one animal (AAV.CAP-B22 injected, 2 years, male, $0.364 \mathrm{~kg}$ ) was found dead ( $29 \mathrm{~d}$ after injection); at necropsy, the pathology report indicated that the death was unrelated to the virus. The day before infusion, the animals' food was removed. Animals were anesthetized with isoflurane in air; the skin over the saphenous vein was shaved and sanitized with an ethanol scrub; and the virus (Extended Data Table 2) was infused over several minutes. Anesthesia was withdrawn, and the animals were monitored until they became active, upon which they were returned to their cages. Activity and behavior were closely monitored over the next $3 \mathrm{~d}$, followed by daily observations thereafter.

DNA/RNA recovery and sequencing. Round 1 and round 2 viral libraries were injected into C57Bl/6J and Cre transgenic animals (Syn1-Cre, GFAP-Cre, Tek-Cre and TH-Cre) at a dose of $8 \times 10^{10}$ viral genomes per animal, and rAAV genomes were recovered 2 weeks after injection, as described in the M-CREATE protocol $^{33}$. To determine the number of variants included in round 2, 0.01 times the enrichment of the top variant in each tissue was set as a threshold, and variants above that threshold were included. Mice were euthanized, and most major organs were recovered, snap-frozen on dry ice and placed into long-term storage at $-80^{\circ} \mathrm{C}$. Tissues collected included brain, spinal cord, DRG, liver, lungs, heart, stomach, intestines, kidneys, spleen, pancreas, testes, skeletal muscle and adipose tissue. Then, $100 \mathrm{mg}$ of each tissue ( $250 \mathrm{mg}$ for brain hemispheres and $<100 \mathrm{mg}$ for DRG) was homogenized in TRIzol (Life Technologies, 15596) using a BeadBug (Benchmark Scientific, D1036), and viral DNA was isolated according to the manufacturer's recommended protocol. Recovered viral DNA was treated with RNase, underwent restriction digestion with SmaI (found within the inverted terminal repeats) to improve later rAAV genome recovery by polymerase chain reaction (PCR) and purified with a Zymo DNA Clean and Concentrator kit (D4033). Viral genomes flipped by Cre recombinase in select transgenic lines (or pre-flipped in WT animals) were selectively recovered using the following primers: $5^{\prime}$-CTTCCAGTTCAGCTACGAGTTTGAGAAC- $3^{\prime}$ and $5^{\prime}$-CAAGTAAAACCTCTACAAATGTGGTAAAATCG-3', after 25 cycles of $98^{\circ} \mathrm{C}$ for $10 \mathrm{~s}, 60^{\circ} \mathrm{C}$ for $15 \mathrm{~s}$ and $72^{\circ} \mathrm{C}$ for $40 \mathrm{~s}$, using Q5 DNA polymerase in five $25-\mu \mathrm{l}$ reactions with $50 \%$ of the total extracted viral DNA as a template.

After Zymo DNA purification, samples from the WT C57Bl/6J animals were serially diluted from 1:10 to 1:10,000, and each dilution was further amplified around the library variable region. This amplification was done using the following primers: 5' -ACGCTCTTCCGATCTAATACTTGTACTATCTCTCTAGAACTATT $-3^{\prime}$ and $5^{\prime}$-TGTGCTCTTCCGATCTCACACTGAATTTTAGCGTTTG-3' ${ }^{\prime}$, after ten cycles of $98^{\circ} \mathrm{C}$ for $10 \mathrm{~s}, 61^{\circ} \mathrm{C}$ for $15 \mathrm{~s}$ and $72^{\circ} \mathrm{C}$ for $20 \mathrm{~s}$, to recover $73 \mathrm{bp}$ of viral genome around and including the 21 -bp variable region and add adapters for Illumina NGS. After PCR cleanup, these products were further amplified using NEBNext Dual Index Primers for Illumina sequencing (New England Biolabs, E7600), after ten cycles of $98^{\circ} \mathrm{C}$ for $10 \mathrm{~s}, 60^{\circ} \mathrm{C}$ for $15 \mathrm{~s}$ and $72^{\circ} \mathrm{C}$ for $20 \mathrm{~s}$. The amplification products were run on a $2 \%$ low-melting-point agarose gel (Thermo Fisher Scientific, 16520050) for better separation and recovery of the 210-bp band. The dilution series was analyzed for each WT tissue and the highest concentration 
that resulted in no product from WT tissue on the gel was chosen for the amplification of the viral DNA from the transgenic animal tissues. This process was performed to differentiate between viral genomes flipped before packaging or due to Cre in the animal. Pre-flipped viral genomes should be avoided to minimize false positives in the NGS results.

All Cre-flipped viral genomes from transgenic animal tissues were similarly amplified (using the dilutions that do not produce pre-flipped viral genomes) to add Illumina sequencing adapters and subsequently for index labeling. The amplified products now containing unique indices for each tissue from each animal were run on a low-melting-point agarose gel, and the correct bands were extracted and purified with a Zymoclean Gel DNA Recovery kit.

Packaged viral library DNA was isolated from the injected viral library by digestion of the viral capsid and purification of the contained ssDNA. These viral genomes were amplified by two PCR amplification steps, like the viral DNA extracted from tissue, to add Illumina adapters and then indices. Correct bands were extracted and purified after gel electrophoresis. This viral library DNA, along with the viral DNA extracted from tissue, was sent for deep sequencing using an Illumina HiSeq 2500 System (Millard and Muriel Jacobs Genetics and Genomics Laboratory, California Institute of Technology).

A pool of eight viruses (AAV9, AAV-PHP.eB, AAV.CAP-B1, AAV.CAP-B2, AAV.CAP-B8, AAV.CAP-B10, AAV.CAP-B18 and AAV.CAP-B22) packaging CAG-FXN-HA with unique 12-bp barcodes were injected into two adult marmosets (Extended Data Table 1). After 6 weeks, animals were euthanized, and brain and liver were recovered and snap-frozen. Then, $1-\mathrm{mm}$ coronal sections from each tissue ( $4 \mathrm{~mm}$ for the brain) were homogenized in TRIzol (Life Technologies, 15596) using a BeadBug (Benchmark Scientific, D1036), and total RNA was recovered according to the manufacturer's recommended protocol. Recovered RNA was treated with DNase, and cDNA was generated from the mRNA using SuperScript III (Thermo Fisher Scientific, 18080093) and oligo(dT) primers according to the manufacturer's recommended protocol. Barcoded FXN transcripts were recovered from the resulting cDNA library, as well as the injected pool, using the following primers: $5^{\prime}$-TGGACCTAAGCGTTATGACTGGAC- $3^{\prime}$ and $5^{\prime}$-GGAGCAACATAGTTAAGAATACCAGTCAATC-3', after 25 cycles of $98^{\circ} \mathrm{C}$ for $10 \mathrm{~s}, 63^{\circ} \mathrm{C}$ for $15 \mathrm{~s}$ and $72^{\circ} \mathrm{C}$ for $20 \mathrm{~s}$, using Q5 DNA polymerase in five reactions using $50 \mathrm{ng}$ of cDNA or viral DNA each as a template. After Zymo DNA purification, samples were diluted 1:100 and further amplified around the barcode region using the following primers: $5^{\prime}$-ACGCTC TTCCGATCTTGTTCCAGATTACGCTTGAG-3' ${ }^{\prime}$ and 5' $^{\prime}$-TGTGCTCTT CCGATCTTGTAATCCAGAGGTTGATTATCG-3', after ten cycles of $98^{\circ} \mathrm{C}$ for $10 \mathrm{~s}, 55^{\circ} \mathrm{C}$ for $15 \mathrm{~s}$ and $72^{\circ} \mathrm{C}$ for $20 \mathrm{~s}$. After PCR cleanup, these products were further amplified using NEBNext Dual Index Primers for Illumina sequencing (New England Biolabs, E7600), after ten cycles of $98^{\circ} \mathrm{C}$ for $10 \mathrm{~s}, 60^{\circ} \mathrm{C}$ for $15 \mathrm{~s}$ and $72{ }^{\circ} \mathrm{C}$ for $20 \mathrm{~s}$. The amplification products were run on a $2 \%$ low-melting-point agarose gel (Thermo Fisher Scientific, 16520050) for better separation and recovery of the 210-bp band. All indexed samples were sent for deep sequencing as before

Individual variants (AAV9, AAV-PHP.eB, AAV.CAP-B10 and AAV.CAP-B22) packaging CAG-FXN-HA with unique 12-bp barcodes were injected into 13 individual adult marmosets (Extended Data Table 2). After between 5 and 6 weeks, animals were euthanized, and brain and liver were recovered and snap-frozen. For DNA extraction from these tissues, 25-mg sections from the cortex and the liver were processed using a QIAamp DNA Mini Kit (Qiagen, 51304) to obtain purified viral and genomic DNA from the samples. For RNA transcript extration, 100-mg sections from the cortex and the liver (taken from consistent sections of tissue across animals) were homogenized in TRIzol (Life Technologies, 15596) using a BeadBug (Benchmark Scientific, D1036), and total RNA was recovered according to the manufacturer's recommended protocol. From purified RNA, cDNA was generated with SuperScript IV VILO MasterMix (Thermo Fisher Scientific, 11766050 ) and oligo (dT) primers according to the manufacturer's recommended protocol (including the DNase step).

Viral genome and RNA transcript copy numbers were determined through qPCR as described ${ }^{59}$ using FXN-HA-specific primers: $5^{\prime}$-GACCTAAG CGTTATGACTGG-3' and 5'-AATCTGGAACATCGTATGGG-3'. Within each sample, the viral genome or transcript copy number was normalized on a per-cell basis by quantifying GAPDH transcripts in each sample using the GAPDH-specific primers: 5' ${ }^{\prime}$ TGTTCCAGTATGATTCCACC- ${ }^{\prime}$ and 5'-GATGACCCTTTTGGCTCC-3'. DNA and RNA in tissues from animals MSV5, MSV6 and MSV7 were analyzed at the SIAT, whereas MPV1-2, MSV1-4 and MSV8-13 were analyzed at the California Institute of Technology.

NGS data alignment and processing. Raw FASTQ files from NGS runs were processed with M-CREATE data analysis code (available on GitHub at https:// github.com/GradinaruLab/mCREATE) that align the data to an AAV9 template DNA fragment containing the 21-bp diversified region between AA452 and AA458, for the two rounds of AAV evolution/selection, or to an FXN-HA template containing the 12-bp unique barcode, for the marmoset virus pool. The pipeline to process these datasets involved filtering to remove low-quality reads, using a quality score for each sequence, and eliminating bias from PCR-induced mutations or high GC content. The filtered dataset was then aligned by a perfect string match algorithm and trimmed to improve the alignment quality. For the AAV engineering, read counts for each sequence were pulled out and displayed along with their enrichment score, defined as the relative abundance of the sequence found within the specific tissue over the relative abundance of that sequence within the injected viral library. For the pooled barcodes, read counts for each sequence were pulled out and normalized to the respective contribution of that barcode to the initial, injected pooled virus to account for small inequalities in the amount of each member of the pool that was injected into the marmosets.

Tissue preparation, immunohistochemistry and immunofluorescence. Mice were euthanized with Euthasol and transcardially perfused with ice-cold $1 \times$ PBS and then freshly prepared, ice-cold $4 \%$ paraformaldehyde (PFA) in $1 \times$ PBS. All organs were excised and post-fixed in $4 \%$ PFA at $4{ }^{\circ} \mathrm{C}$ for $48 \mathrm{~h}$ and then sectioned at $50 \mu \mathrm{m}$ with a vibratome. Immunofluorescence was performed on floating sections with primary and secondary antibodies in PBS containing 10\% donkey serum and $0.1 \%$ Triton X-100. Primary antibodies used were rabbit anti-NeuN (1:200, Abcam, 177487), rabbit anti-S100 (1:200, Abcam, 868), rabbit anti-Olig2 (1:200, Abcam, 109186) and rabbit anti-Calbindin (1:200, Abcam, 25085). Primary antibody incubations were performed for $16-20 \mathrm{~h}$ at room temperature. The sections were then washed and incubated with secondary Alexa Fluor 647-conjugated anti-rabbit FAB fragment antibody (1:200, Jackson ImmunoResearch, 711-607-003) for 6-8 h at room temperature. For nuclear staining, floating sections were incubated in PBS containing $0.2 \%$ Triton X-100 and DAPI (1:1,000, Sigma-Aldrich, 10236276001) for $6-8 \mathrm{~h}$ and then washed. Stained sections were then mounted with ProLong Diamond Antifade Mountant (Thermo Fisher Scientific, P36970).

Marmosets were euthanized (Euthanasia, VetOne) and perfused with $1 \times$ PBS. One hemisphere of the brain (cut into coronal blocks) and the liver were flash-frozen in 2-methylbutane (Sigma-Aldrich, M32631) chilled with dry ice. The other hemisphere and organs were removed and post-fixed with $4 \%$ PFA at $4{ }^{\circ} \mathrm{C}$ for $48 \mathrm{~h}$. These organs were then cryoprotected using $10 \%$ glycerol followed by $20 \%$ glycerol and flash-frozen in 2-methylbutane chilled with dry ice. The blocks of tissue were sectioned on an AO sliding microtome, except for spinal cord and DRG, which were cryosectioned. Then, $50-\mu \mathrm{m}$ slices $(20-\mu \mathrm{m}$ for spinal cord and DRG) were collected in PBS, and immunohistochemistry or immunofluorescence was performed on floating sections.

To visualize cells expressing the HA-tagged FXN from the variant pool, slices were incubated overnight at room temperature with a rabbit anti-HA primary antibody (1:200, Cell Signaling Technologies, C29F4). After primary incubation, sections were washed in PBS and then incubated with a biotinylated goat anti-rabbit secondary antibody (1:200, Vector Laboratories, BA1000) for $1 \mathrm{~h}$ at room temperature. Sections were again washed in PBS and incubated for $2 \mathrm{~h}$ in ABC Elite (Vector Laboratories, PK6100) as outlined by the supplier. The ABC peroxidase complex was visualized using 3,3'-diaminobenzidine tetrahydrochloride hydrate (Sigma-Aldrich, D5637) for $5 \mathrm{~min}$ at room temperature. Sections were then mounted for visualization.

To visualize cells expressing the HA-tagged FXN for individual variant injections, immunofluorescence staining was performed on floating sections with primary and secondary antibodies in PBS containing 10\% donkey serum and $0.1 \%$ Triton X-100. Primary antibodies used were rat anti-HA (1:200, Roche, $3 F 10)$, rabbit anti-NeuN (1:200, Abcam, 177487) and rabbit anti-S100 beta (1:200, Abcam, 52642). Primary antibody incubations were performed for $16-20 \mathrm{~h}$ at room temperature. The sections were then washed and incubated with secondary anti-rat Alexa Fluor 488 (1:200, Thermo Fisher Scientific, A-21208) and anti-rabbit Alexa Fluor 647 (1:200, Thermo Fisher Scientific, A32795). Stained sections were then washed with PBS and mounted with ProLong Diamond Antifade Mountant.

\section{Imaging and quantification. All CAG-mNeonGreen-expressing tissues were} imaged on a Zeiss LSM 880 confocal microscope using a Fluar $\times 50.25$ M27 objective, with matched laser powers, gains and gamma across all samples of the same tissue. The acquired images were processed in Zen Black 2.3 SP1 (Zeiss)

All CAG-NLS-GFP-expressing tissues were imaged on a Keyence BZ-X all-in-one fluorescence microscope at 48-bit resolution with the following objectives: PlanApo- $\lambda \times 20 / 0.75$ ( $1 \mathrm{~mm}$ working distance) or PlanApo- $\lambda \times 10 / 0.45$ ( $4 \mathrm{~mm}$ working distance). For co-localization of GFP expression to antibody staining, in some cases the exposure time for the green (GFP) channel was adjusted to facilitate imaging of high- and low-expressing cells while avoiding oversaturation. In all cases in which fluorescence intensity was compared between samples, exposure settings and changes to gamma or contrast were maintained across images. To minimize bias, multiple fields of view per brain region and peripheral organ were acquired for each sample. For brain regions, the fields of view were matched between samples and chosen based on the antibody staining rather than GFP signal. For peripheral tissues, fields of view were chosen based on the DAPI or antibody staining to preclude observer bias.

Marmoset tissue sections transduced with the variant pool were examined and imaged on a Zeiss AxioImager Z1 with an Axiocam 506 color camera. Acquired images were processed in Zen Blue 2 (Zeiss).

Marmoset tissues transduced with individual variants were imaged on a Zeiss LSM 880 confocal microscope. Tissues from animals MSV5, MSV6 and MSV7 were imaged at the SIAT, whereas all other marmosets were imaged at the 
California Institute of Technology. Whole tissue sections were imaged using a Fluor $\times 50.25 \mathrm{M} 27$ objective with a gallium arsenide phosphide photomultiplier tube detector at a pixel size of $1.25 \mu \mathrm{m} \times 1.25 \mu \mathrm{m}$. Images for cortex quantification were imaged with a LD-LCI Plan-Apochromat $\times 10 / 0.45$ M27 objective with a photomultiplier tube detector at a pixel size of $0.42 \mu \mathrm{m} \times 0.42 \mu \mathrm{m}$. Images for liver quantification were taken with an LD-LCI Plan-Apochromat $\times 10 / 0.45$ M27 objective with a photomultiplier tube detector at a pixel size of $0.42 \mu \mathrm{m} \times 0.42 \mu \mathrm{m}$ or an LD-LCI Plan-Apochromat $\times 20 / 0.8$ M27 with a gallium arsenide phosphide photomultiplier tube detector at a pixel size of $0.156 \mu \mathrm{m} \times 0.156 \mu \mathrm{m}$ (MSV5, MSV6 and MSV7). Imaging at each magnification was performed with matched laser powers, gains and gamma across all samples of the same tissue imaged in the same location, whereas images taken in different locations were matched by maximizing the range indicator. The acquired images were processed in Zen Black 2.3 SP1

(Zeiss). Quantification of transgene expression in marmoset cortex was performed through manual cell counting of co-localized $\mathrm{HA}^{+}$cells with $\mathrm{NeuN}^{+}$staining in maximum intensity projection images. Quantification of transgene expression in the marmoset liver was performed through manual cell counting of co-localized $\mathrm{HA}^{+}$cells with DAPI staining in maximum intensity projection images.

All image processing was performed with the Keyence BZ-X Analyzer (version 1.4.0.1). Co-localization between the GFP signal and antibody or DAPI staining was performed using the Keyence BZ-X Analyzer with the hybrid cell count automated plugin. Automated counts were validated and routinely monitored by comparison with manual hand counts and found to be below the margin of error for manual counts.

To compare total cell counts and fluorescence intensity throughout the brain between samples, an entire sagittal section located 1,200 $\mu \mathrm{M}$ from the midline was imaged using matched exposure conditions with the Keyence BZ-X automated $\mathrm{XY}$ stitching module. Stitched images were then deconstructed in the Keyence BZ-X Analyzer suite and run through the hybrid cell count automated plugin to count the total number of cells in the entire sagittal section. Average fluorescence intensity was calculated by creating a mask of all GFP-positive cells throughout the sagittal section and measuring the integrated pixel intensity of that mask. The total integrated pixel intensity was divided by the total cell count to obtain the fluorescence intensity per cell. In all cases where direct comparisons of fluorescence intensity were made, exposure settings and post-processing contrast adjustments were matched between samples.

Statistics and reproducibility. For the initial characterization of brain and liver expression in mice (Fig. 1d), the experiment was repeated with $n=3$. For the statistical analysis in mice and related graphs (Fig. 2 and Extended Data Figs. $2-4$ ), a single data point was defined as two tissue sections per animal, with multiple technical replicates per section when possible. Technical replicates were defined as multiple fields of view per section, with the following numbers for each region or tissue of interest: cerebellum $=3$, cortex $=4$, hippocampus $=3$, midbrain $=1$, striatum $=3$, thalamus $=4$, liver $=4$, spleen $=2$, testis $=2$, kidney $=2$, lung $=2$, spine $=1, \mathrm{DRG}=1$ and whole sagittal $=1$. Unless otherwise noted, all experimental groups were $n=6$, determined using preliminary data and experimental power analysis. Normality was tested to ensure that the data matched the assumptions of the statistical tests used.

For the initial pooled experiments in marmosets (Fig. 3), the experiment was repeated in two separate animals (Extended Data Table 1). For the statistical analysis in marmoset and related graphs (Fig. 4 and Extended Data Figs. 5 and 6), a single data point was defined as a single tissue section from a single animal. The data were collected across four separate cohorts in three separate locations (Extended Data Table 2), and the trend was recapitulated in each. For representative brain images and quantification (Fig. 4a,b and Extended Data Fig. $6 \mathrm{~b}$ ), the experiment was repeated $n$ times, where $n=2$ (AAV9), $n=3$ (AAV.PHP. eB), $n=4$ (AAV.CAP-B10) and $n=4$ (AAV.CAP-B22), except for astrocyte staining (Extended Data Fig. 5), which was repeated $n=2$ (AAV9), $n=3$ (AAV.CAP-B10) and $n=2$ (AAV.CAP-B22). For representative liver images and quantification (Fig. $4 \mathrm{c}, \mathrm{d}$ and Extended Data Fig. 6a), the experiment was repeated $n$ times, where $n=1$ (AAV9), $n=3$ (AAV.PHP.eB), $n=2$ (AAV.CAP-B10) and $n=3$ (AAV.CAP-B22). Global brain analysis of AAV.CAP-B10 nervous system expression (Fig. $4 \mathrm{e}, \mathrm{f}$ ) was performed with $n=2$. No statistical methods were used to predetermine sample sizes, but our sample sizes were similar to those reported in our previous publications ${ }^{34,54}$. Data distribution was assumed to be normal, but this was not formally tested.

No data were excluded from analysis. Allocation of organisms and samples to separate groups was random, and animals were allocated to experimental conditions based on availability in each cohort. The investigators were not blinded to allocation during experiments and outcome assessment.

Microsoft Excel for Microsoft 365 (version 2107) and GraphPad Prism 8 for Windows (version 8.4 .3 (686)) were used for statistical analysis and data representation. For all statistical analyses, significance is represented as ${ }^{\star} P \leq 0.05$, ${ }^{* *} P \leq 0.01,{ }^{* * *} P \leq 0.001$ and ${ }^{* * *} P \leq 0.0001$; not significant, $P \geq 0.05$.

Reporting Summary. Further information on research design is available in the Nature Research Reporting Summary linked to this article.

\section{Data availability}

The NGS datasets for capsid selection and marmoset pooled screening that are reported in this article are available under Sequence Read Archive accession code PRJNA769435. The following vector plasmids were deposited in Addgene for distribution, and viruses might be available for commonly packaged genomes (http://www.addgene.org): AAV.CAP-B10 (Addgene, 175004) and AAV.CAP-B22 (Addgene, 175005). All other constructs and tools will be available through the Beckman Institute CLOVER Center (https://clover.caltech.edu/). The data that support the findings of this study are available from the corresponding author upon reasonable request.

\section{Code availability}

The codes used for M-CREATE data analysis were published previously ${ }^{33}$ and are available on GitHub: https://github.com/GradinaruLab/mCREATE.

\section{References}

59. Challis, R. C. et al. Systemic AAV vectors for widespread and targeted gene delivery in rodents. Nat. Protoc. 14, 379-414 (2019).

\section{Acknowledgements}

We wish to thank the entire Gradinaru laboratory for helpful discussions. We thank B. Deverman and S. Kumar for helpful discussions on M-CREATE design and implementation, as described in Kumar et al. ${ }^{33}$. We thank M. Borsos, A. Hori, T. Yan, $\mathrm{X}$. Qu and Y. Chen for their technical assistance. We are grateful to I. Antoshechkin and the Millar and Muriel Jacobs Genetics and Genomics Core at the California Institute of Technology for assistance with next-generation sequencing. We thank M. Smith for helpful discussions and assistance with the bioinformatics pipeline. This work was primarily supported by Defense Advanced Research Projects Agency grant W911NF-17-2-0036 to V.G. and grants from the National Institutes of Health (NIH) to V.G.: Director's New Innovator and PECASE DP2NS087949, BRAIN R01MH117069, BRAIN U01 UMH128336A and NIH Pioneer DP1OD025535. D.G was supported by the National Sciences and Engineering Research Council of Canada. Additional support for revision experiments was provided by K. L. Yang and the Hock E. Tan Center for Molecular Therapeutics in Neuroscience at MIT to G.F., the James and Patricia Poitras Center for Psychiatric Disorders Research at MIT to G.F., NIH grant U24 OD026638 to G.F., Guangdong Provincial Fund for Basic and Applied Basic Research (2019B1515130004) to Y.C., the Shenzhen Knowledge Innovation Program (JCYJ20170413165053031) to Y.C., the National Key R\&D Program of China (2018YFE0203600) to Y.C., the Areas of Excellence Scheme of the University Grants Committee of Hong Kong (AoE/M-604/16) to N.Y.I. and the Hong Kong Center for Neurodegenerative Diseases (INNOHK18SC01) to N.Y.I. In addition, this work is funded by the Beckman Institute at the California Institute of Technology, the Vallee Foundation and the Moore Foundation. V.G. is a Heritage Principal Investigator supported by the Heritage Medical Research Institute. The funders had no role in study design, data collection and analysis, decision to publish or preparation of the manuscript.

\section{Author contributions}

D.G., N.C.F and N.G. analyzed all data and prepared all figures, with input from V.G. N.C.F and N.G. designed and performed the variant selection experiments, characterized the variants in mice and prepared the virus for the pooled marmoset experiments. J.P and A.C. performed National Institutes of Health (NIH) marmoset experiments and analyzed the associated data. J.P. supervised the NIH experiments and helped prepare the associated figure. During revision, the Massachusetts Institute of Technology (MIT), the Shenzhen Institute of Advanced Technology (SIAT) and the Hong Kong University of Science and Technology (HKUST) provided additional marmoset data on controls (AAV-PHP.eB) and to increase ' $n$ ' for all cohorts. D.G. and M.C. prepared the virus for single-variant characterization and performed marmoset single-variant characterization tissue analysis of MIT and NIH marmoset tissues. Q.Z. and J.S. assisted with the MIT AAV injections, animal perfusion and tissue sample collection, under the supervision of G.F. Yijing Chen and Y.F. performed single-variant characterization of the SIAT animals and analyzed the associated data, under the supervision of Yu Chen. L.W. supervised the marmoset breeding at the SIAT. Y.D. coordinated with D.G. for experiments at the SIAT, under the supervision of N.Y.I. N.C.F., N.G., D.G. and V.G. wrote the manuscript, with input from all authors. No experimental work was performed during the Los Angeles COVID-19 stay-at-home order. V.G. supervised all aspects of the work.

\section{Competing interests}

The California Institute of Technology has filed and licensed patent applications for the work described in this manuscript, with N.C.F., N.G. and V.G. listed as inventors (US Patent application no. 16/582,635, 2020). V.G. is a co-founder and board member and N.C.F. and N.G. are co-founders and employees of Capsida Biotherapeutics, a fully integrated AAV engineering and gene therapy company. Capsida Biotherapeutics did not provide funding and had no role in study design, data collection and analysis, decision to publish or preparation of the manuscript. The remaining authors declare no competing interests. 


\section{Additional information}

Extended data is available for this paper at https://doi.org/10.1038/s41593-021-00969-4.

Supplementary information The online version contains supplementary material available at https://doi.org/10.1038/s41593-021-00969-4.
Correspondence and requests for materials should be addressed to Viviana Gradinaru. Peer review information Nature Neuroscience thanks Subhojit Roy and the other, anonymous, reviewer(s) for their contribution to the peer review of this work.

Reprints and permissions information is available at www.nature.com/reprints. 


\section{Amino acid enrichment in brain}

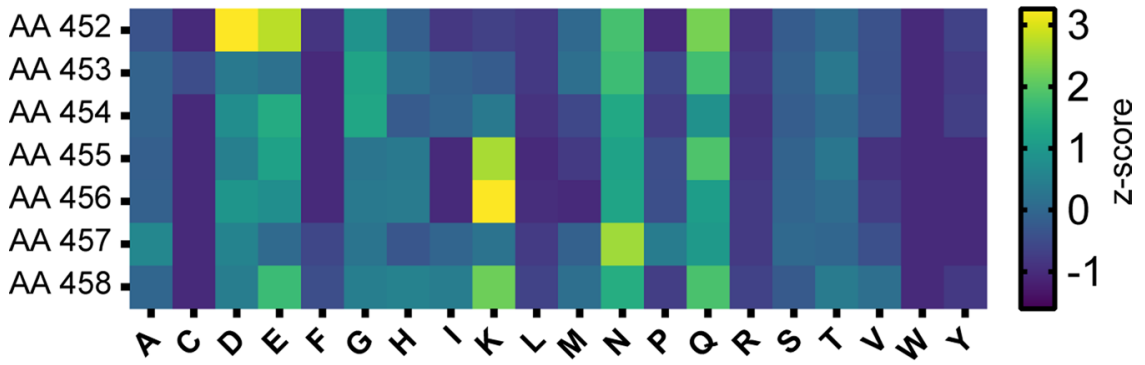

Amino acid enrichment detargeted from liver

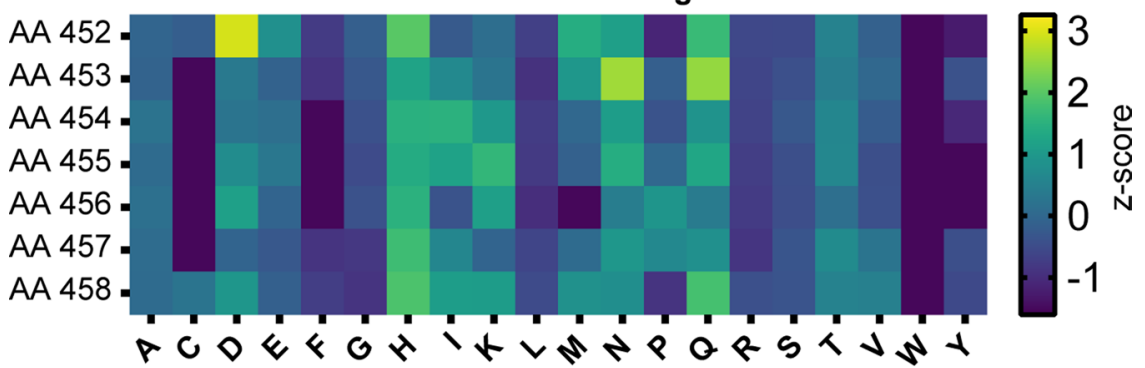

Amino acid enrichment in brain and detargeted from liver

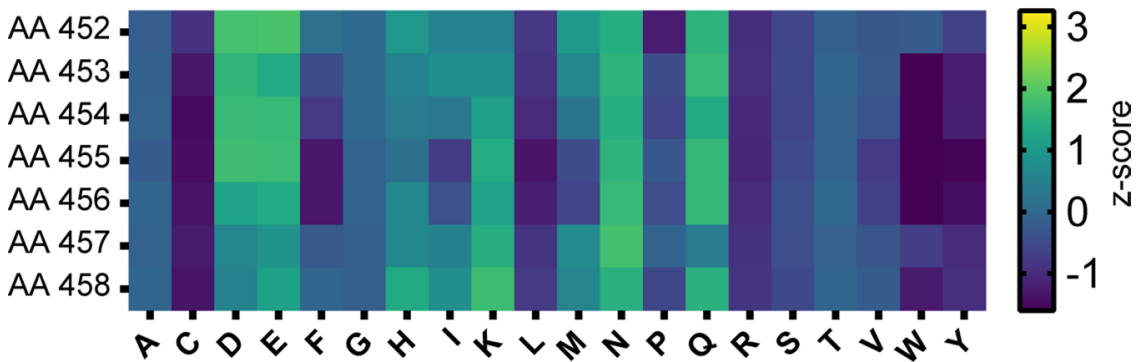

Extended Data Fig. 1 | Amino-acid contributions across the substitution library. (Related to Fig. 1.). Amino-acid contributions across the 7-mer substitution to variants enriched in the brain and targeted away from the liver. The $1 \mathrm{k}$ variants with the highest enrichment in the brain of hSyn-Cre animals, the $1 \mathrm{k}$ variants with the lowest enrichment in the liver of Tek-Cre animals, and all 39,034 variants with positive enrichment in the brain and negative enrichment in the liver from round 2 were analyzed. Plotted is the $z$-score of all amino acids at each position. Amino acids found at a higher prevalence than average are displayed in yellow and those found at a lower prevalence are displayed in purple. 


\section{Spinal cord}

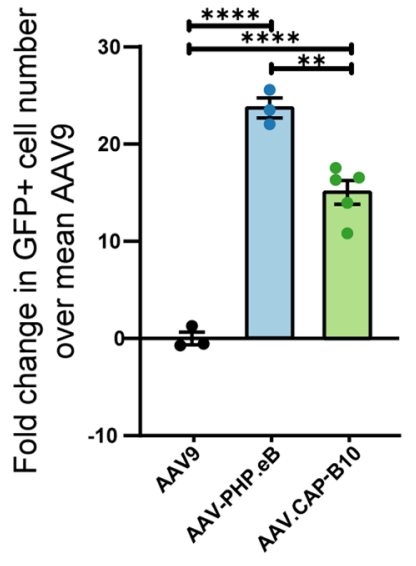

Submucosal plexus
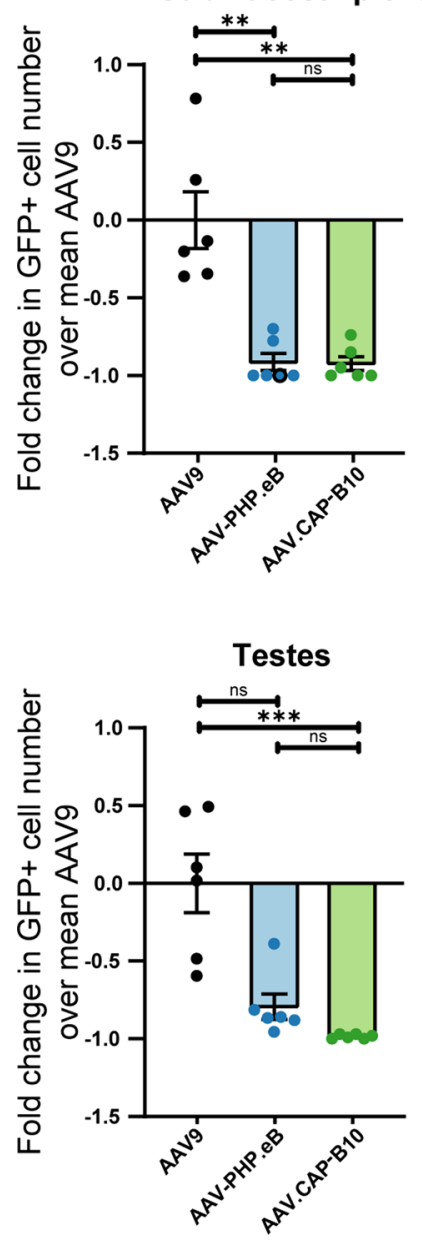

Extended Data Fig. 2 | See next page for caption.

\section{DRGs}
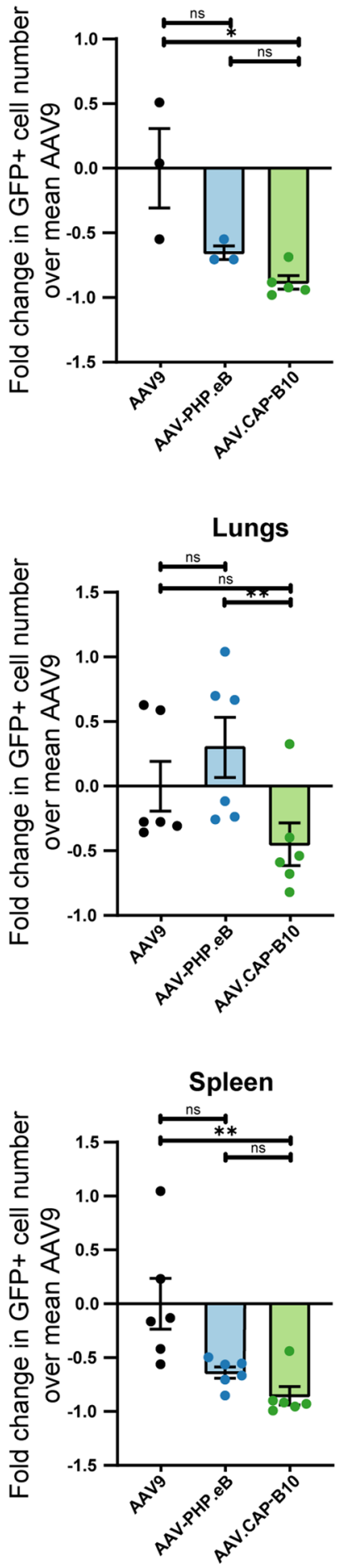

Myenteric plexus

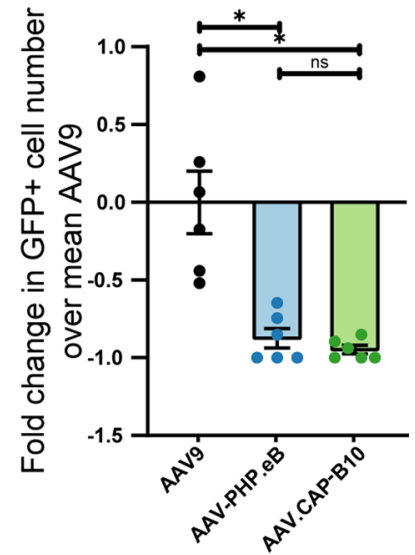

Kidneys
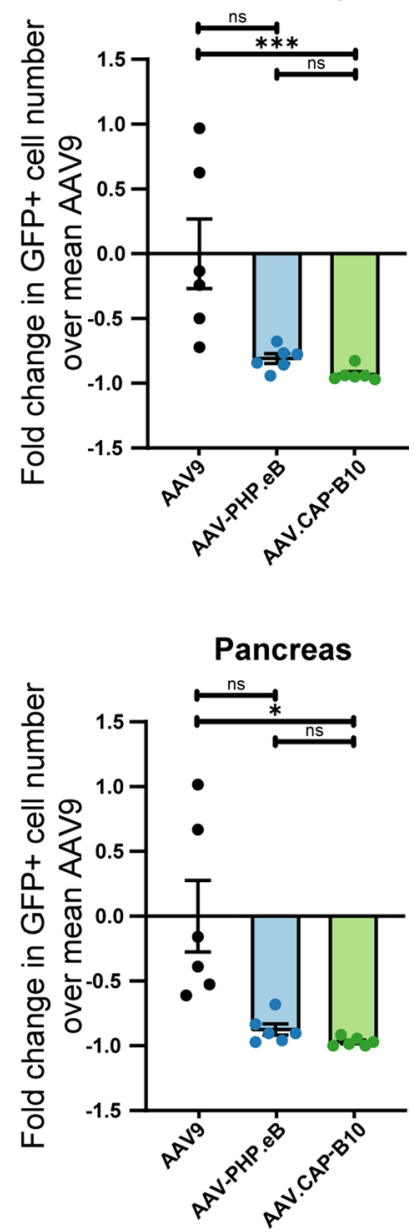
Extended Data Fig. 2 | Quantification of decreased targeting from non-CNS organs in mice. (Related to Fig. 2.). sSAAV9:CAG-NLSx2-EGFP, ssAAV-PHP. eB:CAG-NLSx2-EGFP, and sSAAV.CAP-B10:CAG-NLSx2-EGFP were intravenously injected into male adult mice at $1 \times 10^{11} \mathrm{vg} / \mathrm{mouse}$. GFP fluorescence was assessed after three weeks of expression. The number of cells expressing GFP after delivery with AAV.CAP-B10 in the spinal cord is significantly increased relative to AAV9 and AAV-PHP.eB ( $p=<0.0001$ [AAV9 vs AAV-PHP.eB], <0.0001 [AAV9 vs AAV.CAP-B10], 0.0016 [AAV-PHP.eB vs. AAV.CAP-B10]). The number of cells expressing GFP after delivery with AAV.CAP-B10 in the DRGs is significantly decreased relative to AAV9 and not significantly different from AAV-PHP.eB ( $p=0.6488$ [AAV9 vs AAV-PHP.eB], 0.0229 [AAV9 vs AAV.CAP-B10], 0.5956 [AAV-PHP.eB vs. AAV.CAP-B10]). In the myenteric and submucosal plexi of the intestines, the number of cells expressing GFP after delivery with AAV.CAP-B10 is significantly decreased relative to AAV9 and not significantly different relative to AAV-PHP.eB (Myenteric Plexi: $p=0.0165$ [AAV9 vs AAV-PHP.eB], 0.0147 [AAV9 vs AAV.CAP-B10], 0.6461 [AAV-PHP.eB vs. AAV.CAP-B10]; Submucosal Plexi: $p=0.0066$ [AAV9 vs AAV-PHP.eB], 0.0095 [AAV9 vs AAV.CAP-B10], >0.9999 [AAV-PHP.eB vs. AAV.CAP-B10]). In the lungs, the number of cells expressing GFP after delivery with AAV.CAP-B10 is significantly decreased relative to AAV-PHP.eB and not significantly different from AAV9 ( $p=0.5286$ [AAV9 vs AAV-PHP.eB], 0.2805 [AAV9 vs AAV.CAP-B10], 0.0073 [AAV-PHP.eB vs. AAV.CAP-B10]). In the kidneys, spleen, pancreas, and testes, the number of cells expressing GFP after delivery with AAV.CAP-B10 transduction efficiency is significantly decreased relative to AAV9 and non-significantly changed relative to AAV-PHP.eB (Kidneys: $p=0.1118$ [AAV9 vs AAV-PHP.eB], 0.0010 [AAV9 vs AAV.CAP-B10], 0.3894 [AAV-PHP.eB vs. AAV.CAP-B10]; Spleen: $p=0.1363$ [AAV9 vs AAV-PHP.eB], 0.0024 [AAV9 vs AAV.CAP-B10], 0.5293 [AAV-PHP.eB vs. AAV. CAP-B10]; Pancreas: $p=0.0677$ [AAV9 vs AAV-PHP.eB], 0.0452 [AAV9 vs AAV.CAP-B10], 0.2085 [AAV-PHP.eB vs. AAV.CAP-B10]; Testes: $p=0.2497$ [AAV9 vs AAV-PHP.eB], 0.0005 [AAV9 vs AAV.CAP-B10], 0.1191 [AAV-PHP.eB vs. AAV.CAP-B10]). $n=6$ mice per group except for the spinal cord and DRGs, which had $n=3$ mice for the AAV9 and AAV-PHP.eB groups and $n=5$ mice for the AAV.CAP-B10 group; mean \pm SE; significance was determined using Ordinary one-way ANOVA with Tukey's correction for spinal cord, Brown-Forsythe and Welch one-way ANOVA tests with Dunnett's T3 correction for myenteric plexus and pancreas, Kruskal-Wallis one-way ANOVA test with Dunn's correction for DRGs, submucosal plexus, lungs, kidneys, spleen, and testes. 


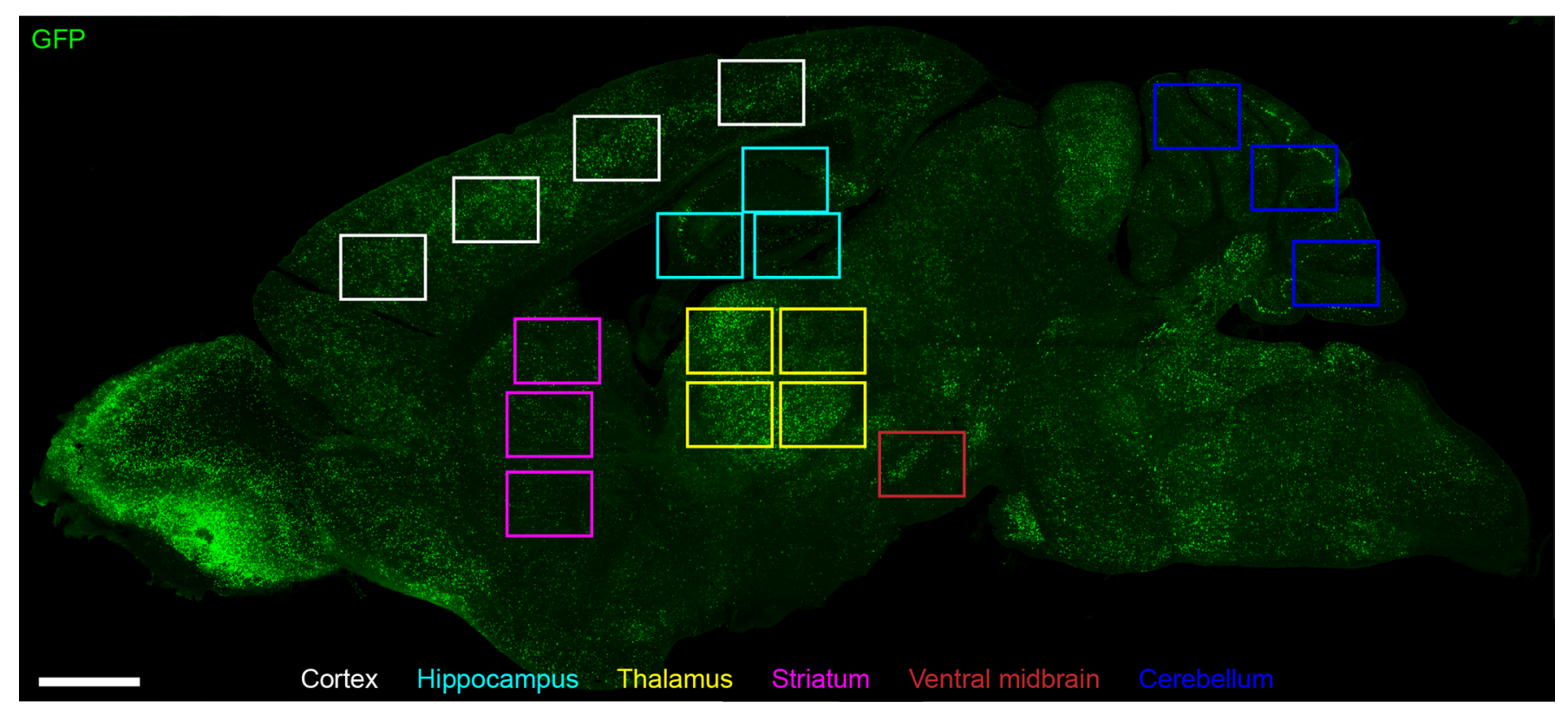

Extended Data Fig. 3 | Brain regions selected for cell-type quantification, depicted on brain expressing NLS-GFP after systemic delivery with AAV-PHP. eB. (Related to Fig. 2.). Four areas of cortex (white), three areas of hippocampus (teal), four areas of thalamus (yellow), three areas of striatum (purple), one area of ventral midbrain (red), and three areas of cerebellum (dark blue) were selected from each brain section quantified. Each section was located roughly $1200 \mu \mathrm{m}$ from the midline for consistency across animals. Scale bar is $1 \mathrm{~mm}$. 


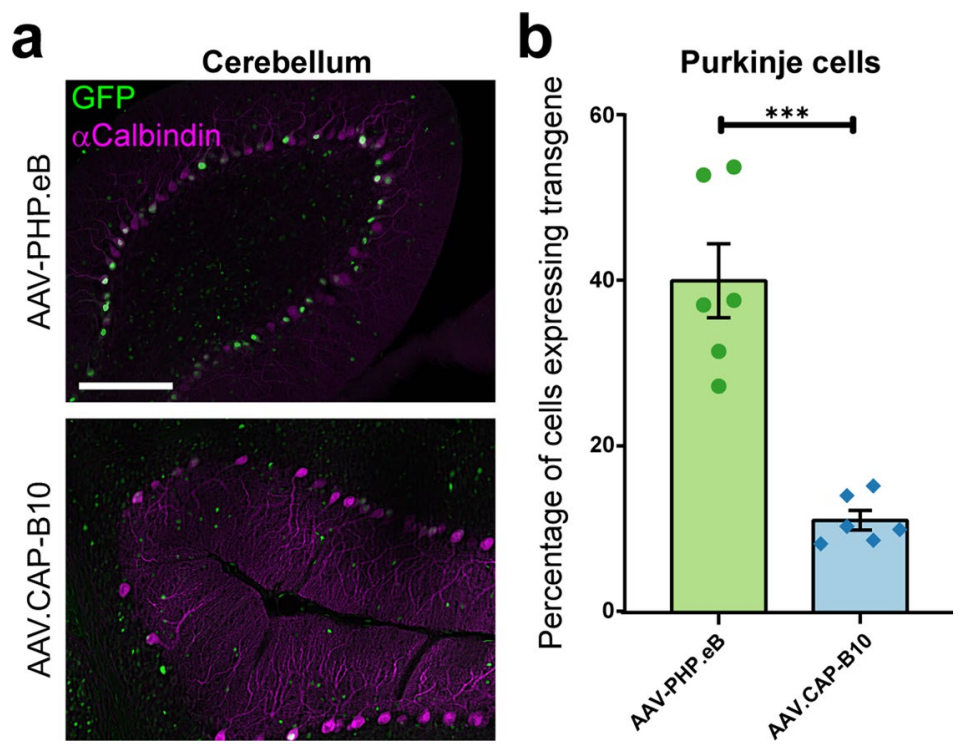

Extended Data Fig. 4 | Quantification of Purkinje cell expression. (Related to Fig. 2.). AAV.CAP-B10 is significantly targeted away from Purkinje cells in the cerebellum. sSAAV-PHP.eB:CAG-NLSx2-EGFP and ssAAV.CAP-B10:CAG-NLSx2-EGFP were intravenously injected into male adult mice at $1 \times 10^{11} \mathrm{vg} /$ mouse. GFP fluorescence was assessed after three weeks of expression. $(\mathbf{a}, \mathbf{b})$ Quantification of the percentage of Purkinje cells displaying GFP expression in the cerebellum shows significantly fewer cells express EGFP after delivery by AAV.CAP-B10 than by AAV-PHP.eB $(P=0.0009)$. $n=6$ mice per group, mean \pm SE, two-tailed Welch's t test. Scale bar is $200 \mu \mathrm{m}$. 

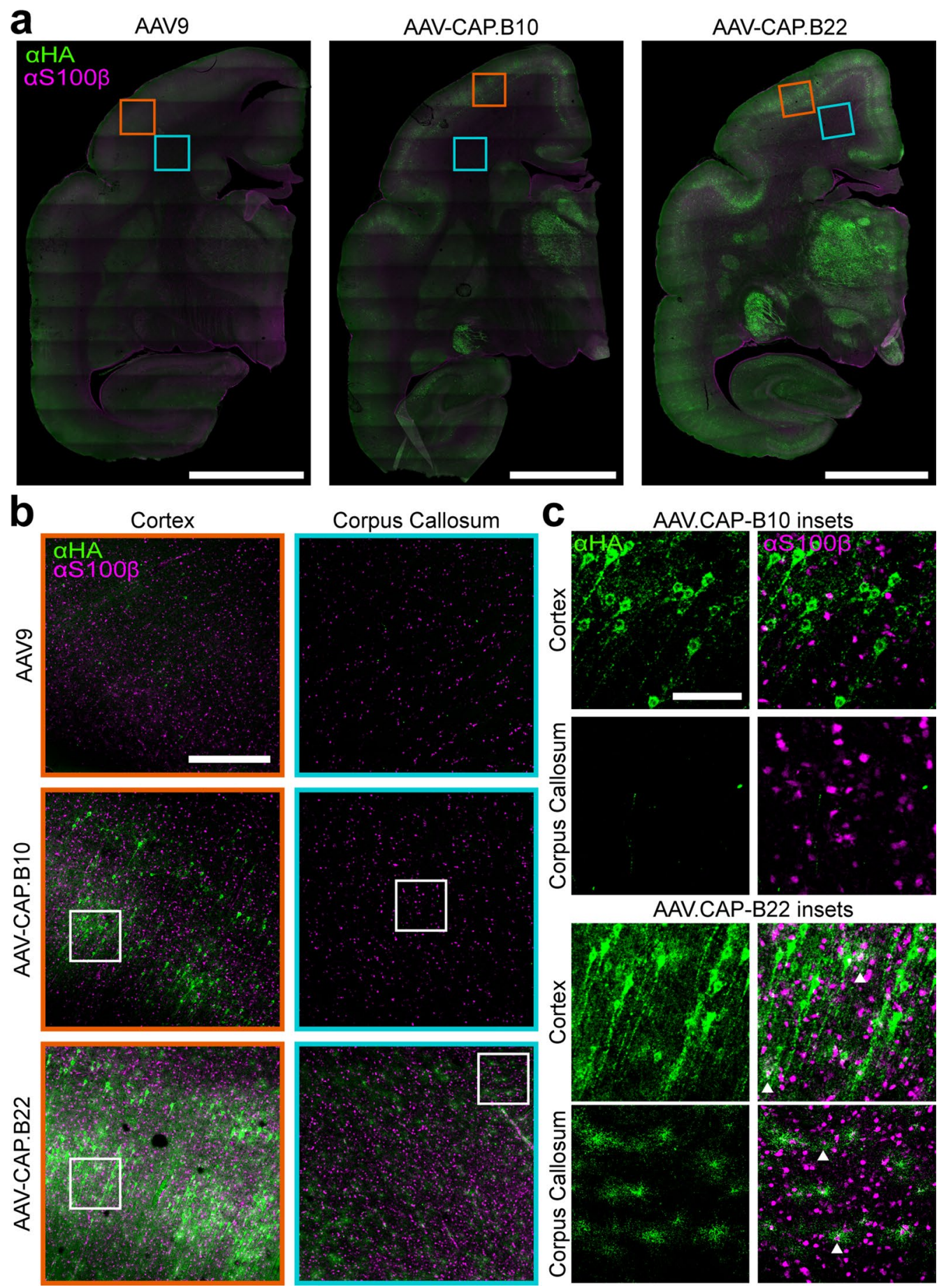

Extended Data Fig. 5 | Astrocyte specificity of AAV9, AAV.CAP-B10, and AAV.CAP-B22 characterized through immunostaining for the HA tag in conjunction with S100B. (Related to Fig. 4.). Astrocyte specificity of AAV9, AAV.CAP-B10, and AAV.CAP-B22 characterized through immunostaining for the HA tag in conjunction with $\mathrm{S100 \beta}$. (a) Coronal sections from marmoset brains after injection of individual variants, showing the locations of the areas selected for magnification. Scale bar is $5 \mathrm{~mm}$. (b) The number of astrocytes expressing FXN was compared in magnified frames taken from the cortex and corpus callosum of animals injected with single variants. AAV9 and AAV.CAP-B10 display very little FXN expression in astrocytes, whereas AAV.CAP-B22 displays FXN expression in astrocytes in both the cortex and the corpus callosum. Scale bar is $500 \mu \mathrm{m}$. (c) Further magnification indicates colocalization (examples marked with arrows) of HA and S100 $\beta$ immunostaining in both the cortex and corpus callosum of marmosets for AAV.CAP-B10 and AAV. CAP-B22. Scale bar is $100 \mu \mathrm{m}$. 
a

Liver Nucleic Acid Quantification

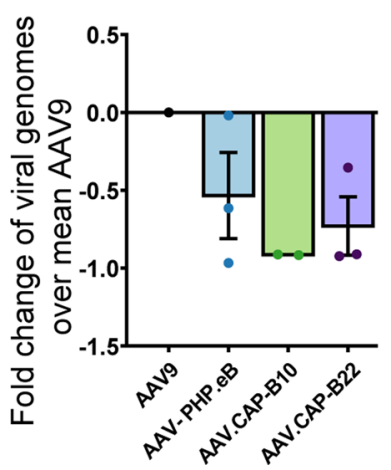

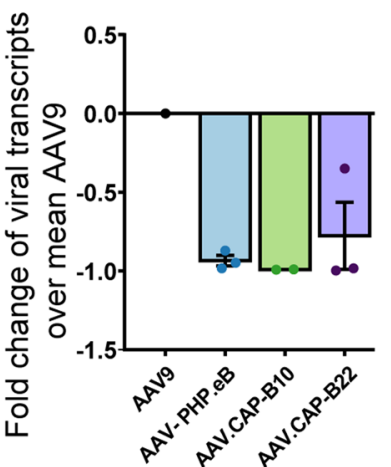

b

Cortex Nucleic Acid Quantification

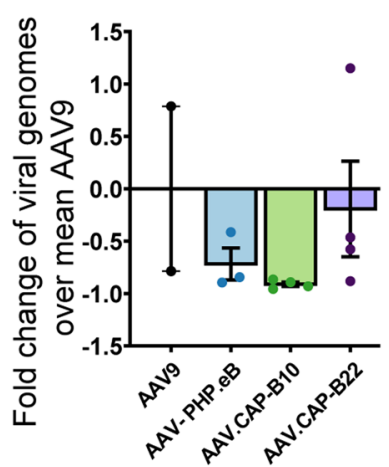

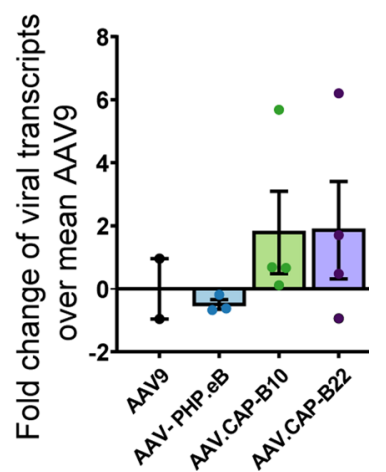

Extended Data Fig. 6 | Viral genome and RNA transcript measurements in cortex and liver tissue after single variant injection in marmosets. (Related to Fig. 4.). (a) Viral genomes and RNA expression measurements in the liver corroborate protein expression data, indicating that AAV.CAP-B10 and AAV.CAP-B22 have decreased transduction and expression in the liver relative to AAV9. $n=1$ for AAV9, 3 for AAV-PHP.eB, 2 for AAV.CAP-B10, 3 for AAV.CAP-B22. mean \pm SE. (b) Viral genome and RNA expression measurements in the cortex indicate no significant difference between experimental conditions. $n=2$ for AAV9, 3 for AAV-PHP.eB, 4 for AAV.CAP-B10, 4 for AAV.CAP-B22, mean \pm SE, two-tailed Welch's t-test. Values for both viral genomes and RNA transcripts were normalized by internal GAPDH control. 


\section{NATURE NEUROSCIENCE}

\section{Extended Data Table 1 | Systemic marmoset injections of a CNS-targeting engineered viral pool}

\begin{tabular}{lllll} 
Marmoset ID & Age (years) & Sex & Weight at injection (kg) & $\begin{array}{l}\text { Dose (viral genomes } \\
\text { per kg) }\end{array}$ \\
\hline MPV1 & 7.6 & Male & 0.386 & $1.2 \times 10^{14}$ \\
MPV2 & 11.5 & Male & 0.375 & 42 \\
& & 42 & $40^{13}$
\end{tabular}


Extended Data Table 2 | Systemic marmoset injections of CNS-targeting engineered viral variants

\begin{tabular}{|c|c|c|c|c|c|c|c|}
\hline Marmoset ID & Location & Age (years) & Sex & $\begin{array}{l}\text { Weight injection } \\
\text { (kg) }\end{array}$ & Variant injected & $\begin{array}{l}\text { Dose (viral genomes } \\
\text { per kg) }\end{array}$ & Length (d) \\
\hline MSV1 & $\mathrm{NIH}$ & 11.5 & Female & 0.300 & AAV9 & $7 \times 10^{13}$ & 34 \\
\hline MSV2 & $\mathrm{NIH}$ & 6.9 & Female & 0.450 & AAV.CAP-B10 & $7 \times 10^{13}$ & 34 \\
\hline MSV3 & $\mathrm{NIH}$ & 12.1 & Male & 0.360 & AAV.CAP-B10 & $7 \times 10^{13}$ & 34 \\
\hline MSV4 & $\mathrm{NIH}$ & 14.0 & Male & 0.400 & AAV.CAP-B22 & $7 \times 10^{13}$ & 34 \\
\hline MSV5 & SIAT & 1.7 & Male & 0.370 & AAV9 & $7 \times 10^{13}$ & 36 \\
\hline MSV7 & SIAT & 2.0 & Male & 0.364 & AAV.CAP-B22 & $7 \times 10^{13}$ & $29^{\star}$ \\
\hline MSV8 & MIT & 2.1 & Male & 0.314 & AAV-PHP.eB & $7 \times 10^{13}$ & 42 \\
\hline MSV9 & MIT & 2.8 & Female & 0.356 & AAV.CAP-B10 & $7 \times 10^{13}$ & 42 \\
\hline MSV10 & MIT & 2.9 & Male & 0.358 & AAV.CAP-B22 & $7 \times 10^{13}$ & 42 \\
\hline MSV11 & $\mathrm{NIH}$ & 6.5 & Male & 0.504 & AAV-PHP.eB & $7 \times 10^{13}$ & 44 \\
\hline
\end{tabular}

Tissue was collected after early death unrelated to the virus. 


\section{Reporting Summary}

Nature Research wishes to improve the reproducibility of the work that we publish. This form provides structure for consistency and transparency in reporting. For further information on Nature Research policies, see our Editorial Policies and the Editorial Policy Checklist.

\section{Statistics}

For all statistical analyses, confirm that the following items are present in the figure legend, table legend, main text, or Methods section.

$\mathrm{n} / \mathrm{a} \mid$ Confirmed

$\bigotimes$ The exact sample size $(n)$ for each experimental group/condition, given as a discrete number and unit of measurement

$\square$ A statement on whether measurements were taken from distinct samples or whether the same sample was measured repeatedly

The statistical test(s) used AND whether they are one- or two-sided

Only common tests should be described solely by name; describe more complex techniques in the Methods section.

$\square$ A description of all covariates tested

$\square$ \ A description of any assumptions or corrections, such as tests of normality and adjustment for multiple comparisons

$\square$ A full description of the statistical parameters including central tendency (e.g. means) or other basic estimates (e.g. regression coefficient)

AND variation (e.g. standard deviation) or associated estimates of uncertainty (e.g. confidence intervals)

For null hypothesis testing, the test statistic (e.g. $F, t, r$ ) with confidence intervals, effect sizes, degrees of freedom and $P$ value noted

Give $P$ values as exact values whenever suitable.

Х $\square$ For Bayesian analysis, information on the choice of priors and Markov chain Monte Carlo settings

Х $\square$ For hierarchical and complex designs, identification of the appropriate level for tests and full reporting of outcomes

\ $\square$ Estimates of effect sizes (e.g. Cohen's $d$, Pearson's $r$ ), indicating how they were calculated

Our web collection on statistics for biologists contains articles on many of the points above.

\section{Software and code}

Policy information about availability of computer code

Data collection Raw fastq files from NGS runs were processed with M-CREATE data analysis code (available on Github at: https://github.com/GradinaruLab/ mCREATE)

Data analysis Graphpad Prism 8.4.1, Keyence BZ-X Analyzer suite 1.4.0.1, Microsoft Excel for Microsoft 365 Version 2107

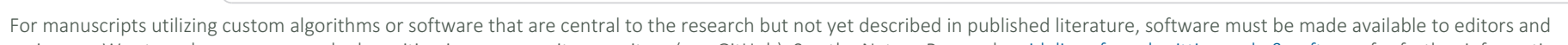

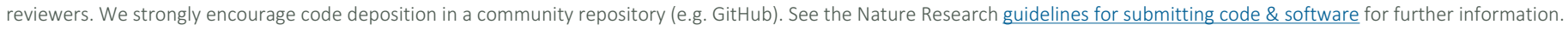

\section{Data}

Policy information about availability of data

All manuscripts must include a data availability statement. This statement should provide the following information, where applicable:

- Accession codes, unique identifiers, or web links for publicly available datasets

- A list of figures that have associated raw data

- A description of any restrictions on data availability

The NGS datasets for capsid selection and marmoset pooled screening that are reported in this article are available under the SRA accession code PRJNA769435. The following vector plasmids are deposited on Addgene for distribution (http://www.addgene.org) AAV.CAP-B10: 175004, AAV.CAP-B22: 175005. The raw data or sequence information is provided in the manuscript, methods section and any other raw data that is not mentioned and that may be necessary to describe our work will be made available upon request. 
Please select the one below that is the best fit for your research. If you are not sure, read the appropriate sections before making your selection.

\ Life sciences

Behavioural \& social sciences

Ecological, evolutionary \& environmental sciences

For a reference copy of the document with all sections, see nature.com/documents/nr-reporting-summary-flat.pdf

\section{Life sciences study design}

All studies must disclose on these points even when the disclosure is negative. $\begin{array}{ll}\text { Sample size } & \begin{array}{l}\text { Sample sizes for mouse work were determined using preliminary data and experimental power analysis. Sample sizes for marmoset data were } \\ \text { based on animal availability and are comparable to previous marmoset literature. }\end{array}\end{array}$

Data exclusions No data was excluded from analysis.

Replication Brain and liver data in Fig.1d was repeated with $n=3$ per viral variant, Characterization of AAV.CAP-B10 in mice (Fig. 2, Extended Data Fig. 2, Extended Data Fig. 3, Extended Data Fig. 4) was repeated with $n=6$ per variant, except in quantification of co-expression of GFP in NeuN+ hippocampal neurons after delivery with AAV.CAP-B10 where $n=3$, and in the spinal cord and DRGs, which had $n=3$ mice for the AAV9 and AAV-PHP.eB groups and $n=5$ mice for the AAV.CAP-B10 group. Experiments of pooled marmoset expression (Fig. 3) were performed with $\mathrm{n}=2$. Individual variant characterization in marmosets was repeated in four separate cohorts at three different locations, with each attempt of replication successful: for individual representative brain images and quantification (Fig. 4a, b, Extended Data Fig. 6b), the experiment was repeated $n$ times, where $n=2$ (AAV9), 3 (AAV.PHP.eB), 4 (AAV.CAP-B10), 4 (AAV.CAP-B22), except for astrocyte staining (Extended Data Fig. 5), which was repeated $n=2$ (AAV9), $n=3$ (AAV.CAP-B10), $n=2$ (AAV.CAP-B22). For representative liver images and quantification (Fig. 4c, $d$, Extended Data Fig. 6a), the experiment was repeated $n$ times, where $n=1$ (AAV9), 3 (AAV.PHP.eB), 2 (AAV.CAP-B10), 3 (AAV.CAP-B22). Global brain analysis of AAV.CAP-B10 nervous system expression (Fig. 4e, f) was performed with $n=2$. All attempts to replicate data were successful.

Randomization Allocation of organisms and samples to separate groups were random.

Blinding The investigators were not blinded during analysis as the differences between control and variant groups were obvious and blinding wouldn't have helped in these experiments.

\section{Reporting for specific materials, systems and methods}

We require information from authors about some types of materials, experimental systems and methods used in many studies. Here, indicate whether each material, system or method listed is relevant to your study. If you are not sure if a list item applies to your research, read the appropriate section before selecting a response.

\begin{tabular}{l|l} 
Materials \& experimental syste \\
\hline $\mathrm{n} / \mathrm{a}$ & Involved in the study \\
\hline & $\bigotimes$ Antibodies \\
$\square$ & $\square$ Eukaryotic cell lines \\
$\square$ & $\square$ Animals and other organisms \\
$\square$ & $\square$ Clinical data \\
\hline
\end{tabular}

\section{Methods} $\mathrm{n} / \mathrm{a}$ Involved in the study

Х $\square$ ChIP-seq

Х $\square$ Flow cytometry

$\triangle \square$ MRI-based neuroimaging

\section{Antibodies}

Antibodies used

In mice, the primary antibodies used were rabbit anti-NeuN (1:200, Abcam, ab177487), rabbit anti-S100 (1:200, Abcam, ab868), rabbit anti-Olig2 (1:200, Abcam, ab109186), rabbit anti-Calbindin (1:200, Abcam, ab25085). The secondary antibodies used were Alexa-647 conjugated anti-rabbit FAB fragment antibody (1:200, Jackson ImmunoResearch Laboratories, Inc., 711-607-003)

For marmoset pooled variant characterization, primary antibodies used were: rabbit anti-HA primary antibody (1:200, Cell Signaling Technologies, C29F4) and goat anti-rabbit secondary antibody (1:200, Vector Laboratories, BA1000)

For marmoset single variant characterization, primary antibodies used were: rat anti-HA (1:200, Roche, 3F10), rabbit anti-NeuN (1:200, Abcam, ab177487), and rabbit anti-S100 beta (1:200, Abcam, ab52642). Secondary antibodies used were: anti-rat Alexa-488 (1:200, ThermoFisher, A-21208) and anti-rabbit Alexa-647 (1:200, ThermoFisher, A32795)

Validation

Abcam, ab177487 (datasheet and citations available): https://www.abcam.com/neun-antibody-epr12763-neuronal-markerab177487.html

Abcam, ab868 (archived datasheet available): https://www.abcam.com/s100-antibody-ab868.html 
Abcam, ab109186 (datasheet and citations available): https://www.abcam.com/olig2-antibody-epr2673-ab109186.html Abcam, ab25085 (archived datasheet available): https://www.abcam.com/calbindin-antibody-ab25085.html

Abcam, ab52642 (datasheet and citations available): https://www.abcam.com/s100-beta-antibody-ep1576y-astrocyte-markerab52642.html

Jackson ImmunoResearch Laboratories, Inc., 711-607-003 (data and citations available): https://www.jacksonimmuno.com/catalog/ products/711-607-003

Cell Signaling Technologies, C29F4 (datasheet and citations available): https://www.cellsignal.com/products/primary-antibodies/hatag-c29f4-rabbit-mab/3724

Vector Laboratories, BA1000 (datasheet and citations available): https://vectorlabs.com/biotinylated-goat-anti-rabbit-iggantibody.html

Roche, 3F10 (datasheet and citations available): https://www.sigmaaldrich.com/catalog/product/roche/roahaha?lang=en\&region=US rabbitData provided in the manuscript.

ThermoFisher, A-21208 (datasheet and citations available): https://www.thermofisher.com/antibody/product/Donkey-anti-Rat-lgGH-L-Highly-Cross-Adsorbed-Secondary-Antibody-Polyclonal/A-21208

ThermoFisher, A32795 (datasheet available): https://www.thermofisher.com/antibody/product/Donkey-anti-Rabbit-lgG-H-L-HighlyCross-Adsorbed-Secondary-Antibody-Polyclonal/A32795

\section{Eukaryotic cell lines}

Policy information about cell lines

Cell line source(s)

293T cells from ATCC

Authentication

No authentication was performed as the source was reliable and we routinely use them in lab, from multiple stocks from ATCC with similar performance.

Mycoplasma contamination

Negative for mycoplasma contamination.

Commonly misidentified lines

(See ICLAC register)

No commonly misidentified cell lines were used in this study.

\section{Animals and other organisms}

Policy information about studies involving animals; ARRIVE guidelines recommended for reporting animal research

Laboratory animals

C57BI/6J WT mice (000664), Syn1-Cre (3966), GFAP-Cre (012886), and Tek-Cre (8863) mice lines used in this study were purchased from the Jackson Laboratory (JAX). Mice were housed under standard conditions between 71 and $75{ }^{\circ} \mathrm{F}, 30 \%-70 \%$ humidity, and light cycle of 13 hours on \& 11 hours off. For round 1 and round 2 selections from the viral library, we used one male and one female mouse from each transgenic line (aged 8-12 weeks), as well as a single male C57BI/6J mouse. Both genders were used for capsid selection in transgenic lines to recover capsid variants with minimal gender bias. For validation of individual viral variants, male C57BI/6J mice aged 6-8 weeks were used. The gender was kept consistent during this validation to avoid discrepancies that exist in the viral expression due to gender differences.

Nine marmosets were born and raised in NIMH. Two of the animals were used for the pooled injection study, both males (ages 7.6, 11.5 years). Seven of the animals were injected with single variants for characterization, but only six were usable (three females and four males, aged 3.5-14 years) as one animal (6.9 years, female) was found dead; at necropsy the pathology report indicated chronic nephritis unrelated to the virus.

Three marmosets were born and raised in Massachusetts Institute of Technology (two males and one female, aged 2.8-6.5 years). All three were injected with single variants for characterization.

Three marmosets were born and raised in Shenzhen Institute of Advanced Technology (all three male, aged 1.7-2.0 years). All three were injected with single variants for characterization.

Wild animals No wild animals were involved in this study.

Field-collected samples The study did not involve any field-collected samples,

Ethics oversight

Mouse procedures performed in this study were approved by the California Institute of Technology Institutional Animal Care and Use Committee (IACUC).

Marmoset procedures at the NIH were approved by ACUC of the National Institutes of Mental Health.

Marmoset procedures at MIT were approved by Committee on Animal Care.

Marmoset procedures at Shenzhen Institute of Advanced Technology were approved by the Institutional Animal Care and Use Committee.

Note that full information on the approval of the study protocol must also be provided in the manuscript. 